\title{
M94 as a Unique Testbed for Black Hole Mass Estimates and AGN Activity at Low Luminosities
}

\author{
Anca Constantin ${ }^{1}$ and Anil C. Seth ${ }^{2}$ \\ ${ }^{1}$ Department of Physics and Astronomy, James Madison University, Harrisonburg, VA 22807, USA \\ ${ }^{2}$ Department of Physics and Astronomy, University of Utah, Salt Lake City, UT 84112, USA \\ Correspondence should be addressed to Anca Constantin, constaax@jmu.edu
}

Received 28 September 2011; Accepted 12 December 2011

Academic Editor: Antonis Georgakakis

Copyright ( 2012 A. Constantin and A. C. Seth. This is an open access article distributed under the Creative Commons Attribution License, which permits unrestricted use, distribution, and reproduction in any medium, provided the original work is properly cited.

\begin{abstract}
We discuss the peculiar nature of the nucleus of M94 (NGC 4736) in the context of new measurements of the broad H $\alpha$ emission from HST-STIS observations. We show that this component is unambiguously associated with the high-resolution X-ray, radio, and variable UV sources detected at the optical nucleus of this galaxy. These multiwavelength observations suggest that NGC 4736 is one of the least luminous broad-line (type 1) LINERs, with $L_{\mathrm{bol}}=2.5 \times 10^{40} \mathrm{erg} \mathrm{s}^{-1}$. This LINER galaxy has also possibly the least luminous broad-line region known $\left(L_{\mathrm{H} \alpha}=2.2 \times 10^{37} \mathrm{erg} \mathrm{s}^{-1}\right)$. We compare black hole mass estimates of this system to the recently measured $\sim 7 \times 10^{6} M_{\odot}$ dynamical black hole mass measurement. The fundamental plane and $M-\sigma^{*}$ relationship roughly agree with the measured black hole mass, while other accretion-based estimates (the M-FWHM $(\mathrm{H} \alpha)$ relation, empirical correlation of $\mathrm{BH}$ mass with high-ionization mid-IR emission lines, and the X-ray excess variance) provide much lower estimates $\left(\sim 10^{5} M_{\odot}\right)$. An energy budget test shows that the AGN in this system may be deficient in ionizing radiation relative to the observed emission-line activity. This deficiency may result from source variability or the superposition of multiple sources including supernovae.
\end{abstract}

\section{Introduction: LINERs and M94}

Most high-mass galaxies are known to host massive black holes $(\mathrm{BH})$, some passively lurking in their centers while others are actively accreting surrounding material (e.g., [1] and references therein). The mechanism that causes a BH's activity to turn on and off is still largely unknown. Understanding the structure of the active galactic nuclei (AGN) at their lowest luminosities is crucial to determining the physical and possibly evolutionary links between the most luminous galaxy centers and the passive ones. However, at low luminosities it is difficult to disentangle the various emission mechanisms that could be concurrently present in galaxy centers. As a consequence, the dominant power source of a large majority of actively line-emitting galaxies remains ambiguous (e.g., [2] for a review).

Diagnostic diagrams (e.g., [3-6]) are relatively successful in separating out bona fide accretion sources (Seyferts) from nuclei whose emission-line activity is mainly powered by young, hot stars ( $\mathrm{H}$ II galaxies), based on emission line ratios.
At least $50 \%$ of the strong line emitters fall easily onto the $\mathrm{H}$ iI locus; however, only less than $10 \%$ are of the Seyfert type $[5,7,8]$. A large fraction of the objects "in between" these two categories, that exhibit relatively low levels of ionization (i.e., low values of $[\mathrm{O} I I I] / H \beta$ ), maintain reasonably strong forbidden line activity (i.e., high values of $[\mathrm{N} \mathrm{II}] / \mathrm{H} \alpha$ ) and are classified as low-ionization nuclear emission regions (LINERs). The reminder are usually called transition objects (Ts). Whether Ts and Ls are powered, at least partly, by accreting BHs, and thus could be called AGN, is a matter of continuous debate [2].

There are some typical emission characteristics that are considered to be particularly good indications that accretion onto a massive $\mathrm{BH}$ is an important, if not the dominant source of ionization in some Ls, and possibly Ts. The detection of broad $\mathrm{H} \alpha$ emission, regardless of its strength or luminosity, is generally considered to be the clue to AGN emission. Some LINERs (and maybe Ts as well) exhibit these features; however, the majority of these systems show only narrow emission, which could be generated by shocks, 
poststarbursts, or other processes unrelated with accretion. Observations outside of the optical wavelengths often reveal AGN signatures in ambiguous and even starburst galaxy nuclei. X-rays are particularly good tracers of accretion; however, they are not efficient in distinguishing AGN at $L_{X} \lesssim 10^{42} \mathrm{erg} \mathrm{s}^{-1}$, where contamination by X-ray binaries can be significant. X-rays are also unlikely to detect heavily absorbed AGN (i.e., Compton thick; $N_{\mathrm{H}}>1.5 \times 10^{24} \mathrm{~cm}^{-2}$ ). On the other hand, mid-IR high-ionization emission lines like [NeV] $\lambda 14.32 \mu \mathrm{m}, 24.32 \mu \mathrm{m}(97.1 \mathrm{eV})$ appear to be a trustworthy indicator of AGN activity [9-11] due to the extreme conditions (i.e., very hard ionizing radiation) required to produce them; because these features have considerably lower optical depth, their detection can also reveal Compton-thick AGN ([12], e.g., NGC 1068). This technique has now been applied to reveal new and large numbers of optically unidentified AGN $[13,14]$, providing thus sensitive improvements on previous AGN censuses. These studies, along with X-ray and radio studies of nearby galaxies (e.g., [15-17]). suggest that a majority of LINERs and a large fraction of transition galaxies might in fact host accreting black holes. The presence of an accreting black hole does not however guarantee that the accretion power is the dominant source of ionization of those galaxy nuclei. A more recent assessment of the energy budget of LINERs by Eracleous et al. [18] argues that in $85 \%$ of LINERs the AGN ionizing photons are not sufficient for producing the observed nebular emission, and thus other power sources are likely to dominate.

Some new potentially powerful insights into the excitation mechanism of the low-luminosity AGN (LLAGN), and in particular the ambiguous sources, come from recent studies of large statistical samples of nearby galaxy nuclei, which reveal a potential $\mathrm{HII} \rightarrow S \rightarrow T \rightarrow$ LINER $\rightarrow$ Passive Galaxies evolutionary sequence in the process of $\mathrm{BH}$ growth within galaxies $[19,20]$. This sequence traces trends in (1) increasing host halo mass, (2) increasing environmental density, (3) increasing central $\mathrm{BH}$ mass and host stellar mass, (4) decreasing $\mathrm{BH}$ accretion rate, (5) aging of the stellar population associated with their nuclei, and (6) decreasing in the amount of dust obscuration, which might translate into a decrease in the amount of material available for star forming or accretion. In this picture, Seyferts and Ts are transition phases between the initial onset of accretion, usually swamped by the star-forming gas and associated dust, which is seen optically as an H II system, and the final phase of accretion observed as LINERs of already massive BHs. While this idea is supported by various other independent observational studies of low-luminosity AGN and starburst galaxies $[21,22]$, along with state-of-the-art hydrodynamical models for the life cycles of the most luminous AGN (e.g., $[23,24])$ it is very probable that not all sources fit into this scenario. It is also important that this evolutionary sequence idea is tested on samples that span a narrow distribution in Hubble types; because most of the $\mathrm{H}$ iss and Ts are relatively late-type disk galaxies that, likely, never experienced a (recent) major merger, it is possible that the trigger of such a sequence is different from the merger that initiates a similar life cycle at high luminosities. Nevertheless, investigating the challenges certain objects bring to this idea is useful for identifying and quantifying the caveats associated with this sequence. These objects may also be at interesting stages in their galaxy evolution.

NGC 4736 (or M94, UGC 7996) is a captivating example of an ambiguous galaxy nucleus, which poses challenges to the general understanding of AGN phenomena, including the above-mentioned sequence. This object is one of the closest $(d=4.3 \mathrm{Mpc}$; [25]) nearly face-on spiral, with a SAab Hubble Type. Its proximity enables study of details that would be unobservable in more distant systems. Its nucleus has a low-luminosity LINER spectrum, but has been also included in catalogs of transition objects [26] or Seyfert 2s (e.g., [27]). The AGN nature of this object has been constantly debated, an aging starburst being a compelling alternative [28-31]. The galaxy presents a ring of $\mathrm{H}$ II regions at a radius of $\sim 50^{\prime \prime}$, red arcs at $\sim 15^{\prime \prime}$, a highsurface-brightness nuclear region, and high far-infrared bulge emission $[32,33]$. Its intricate structure of off-nuclear compact source detections in X-ray [30], radio [31], and UV [29], that do not necessarily match with each other, certainly increases the ambiguity associated with the nature of the main nuclear ionization mechanism. A common implied scenario in all of these studies is that this system is probably in the final stages of a merger.

We reexamine here NGC 4736 in the context of additional evidence for its AGN nature, which is the detection of a broad $\mathrm{H} \alpha$ component in its nuclear spectrum, as observed by the Hubble Space Telescope (HST) with the Space Telescope Imaging Spectrograph (STIS). We also gather multiwavelength data and show that source of the broad $\mathrm{H}$-alpha emission line is coincident with a compact $\mathrm{X}$-ray and radio source. These observations suggest that NGC 4736 hosts a broad-line region of significantly low luminosity, which makes this object one of the least luminous LINERs with strong evidence for $\mathrm{BH}$ accretion. Interestingly, $\mathrm{BH}$ mass indicators calibrated on rapidly accreting Seyfert galaxies give highly discrepant mass estimates in this more quiescent system. The $\sim 10^{7} M \odot$ value given by the $M-\sigma^{*}$ is two orders of magnitude higher than the values obtained via estimators based on the observed emission (X-ray variability, scaling relations, mid-IR emission) which, although physically independent of each other, give a consistent result of $\sim 10^{5} \mathrm{M}_{\odot}$. We are thus facing the following conundrum: either (a) the standard AGN BH mass indicators do not necessarily apply to sources emitting in this low-luminosity regime as, probably, the emission mechanism is fundamentally different from that associated with higher-luminosity AGN, or (b) the emission signatures do not trace accretion onto the central $\mathrm{BH}$. We discuss possible resolutions of this discrepancy in Section 5, where we propose some rather exotic scenarios.

\section{Data Compilation and Analysis}

NGC 4736 has been quite extensively observed across the whole electromagnetic spectrum. We present in this section the multiwavelength observations of this galaxy nucleus, in connection to new measurements of the broad $\mathrm{H} \alpha$ emission detected with HST-STIS. 
2.1. The Broad $\mathrm{H} \alpha$ Emission Observed with HST-STIS. Highresolution optical spectra of the NGC 4736 nucleus were obtained with HST-STIS on July 2002. Data are publicly available (Prop ID 8591) but have not been published. The observations were obtained with the $52^{\prime \prime} \times 0 .{ }^{\prime \prime} 1$ aperture oriented at $\mathrm{PA}=49.65^{\circ}$, with the slit centered along the major axis of the starlight distribution; two cosmic-ray split exposures were obtained, one being slightly shifted in the slit direction. The total combined exposure time is $\sim 4000 \mathrm{~s}$. The G750M grating was set at $6581 \AA$, with a scale of $0 .{ }^{\prime \prime} 05 /$ pixel, with no binning. We reduced the spectra using IRAF (IRAF is distributed by NOAO, which is operated by AURA Inc., under contract with the National Science Foundation) and the STIS reduction pipeline maintained by the Space Telescope Science Institute [34]. This reduction included image combination and cosmic-ray rejection, flux calibration, and correction of the wavelength to the heliocentric frame. To measure the nuclear nebular line-emission properties we extracted the 1-dimensional aperture spectrum five pixels wide ( $\left.0 .{ }^{\prime \prime} 25\right)$ centered on the continuum peak. The extracted spectrum thus consists of the central emission convolved with the STIS spatial point-spread function (PSF) and sampled over a rectangular aperture of 0. .' $^{\prime} 5 \times 0$. . $^{\prime} 1$. The measurements span $6295-6867 \AA$ with a resolution of $0.87 \AA$ $\left(\sigma_{\text {inst }}=17 \mathrm{~km} \mathrm{~s}^{-1}\right)$.

Figure 1 shows the resulting spectrum together with the best matching continuum model, spectral fits of the pure emission-line component, and the associated residuals. The continuum model of the underlying stellar population is obtained via a $\chi^{2}$ minimization of a nonnegative leastsquares fit between the observed spectrum and a sum of discrete star bursts of different ages, adopted from the Bruzual and Charlot [35] stellar population synthesis templates, together with dust attenuation modeled as an additional free parameter. The continuum fitting is performed using an adaptation to our data of Christy Tremonti's code [36]. The pure emission-line spectrum, obtained by subtracting the modeled continuum from the observed spectrum, is fit by a combination of linear continuum and Gaussian components. In addition to narrow emission, the $\mathrm{H} \alpha+$ [NII] feature shows clear evidence for a broader feature. A flux ratio of $1: 3$ was assumed for the [N II] doublet, as dictated by the branching ratio [37]; the [O I] feature and the [N II] and [S II] doublets were assumed to share common velocity (red) shifts and widths. The best-fitting Gaussian parameters were derived via an interactive $\chi^{2}$ minimization, using SPECFIT [38]. Because of the generally low signal to noise of the 2-d spectrum we are not able to test whether our measurements of the broad $\mathrm{H} \alpha$ feature could be corrupted by a possible rotating disk as in the case of M84 (e.g., [39]); the match in the widths of the [S II] and [N II] narrow features (as long as that of the narrow $\mathrm{H} \alpha$ ) argue however against such a significant effect.

Measurements related to the broad $\mathrm{H} \alpha$ feature from the STIS measurements are listed in Table 1 . The fractional contribution of this broad component to the total flux of the $\mathrm{H} \alpha+[\mathrm{NII}]$ blend is $80 \%$. Compared to other galaxies with broad-line emission in the Palomar survey [40], the width of the broad $\mathrm{H} \alpha$ is typical $\left(1570 \mathrm{~km} \mathrm{~s}^{-1}\right)$. However,

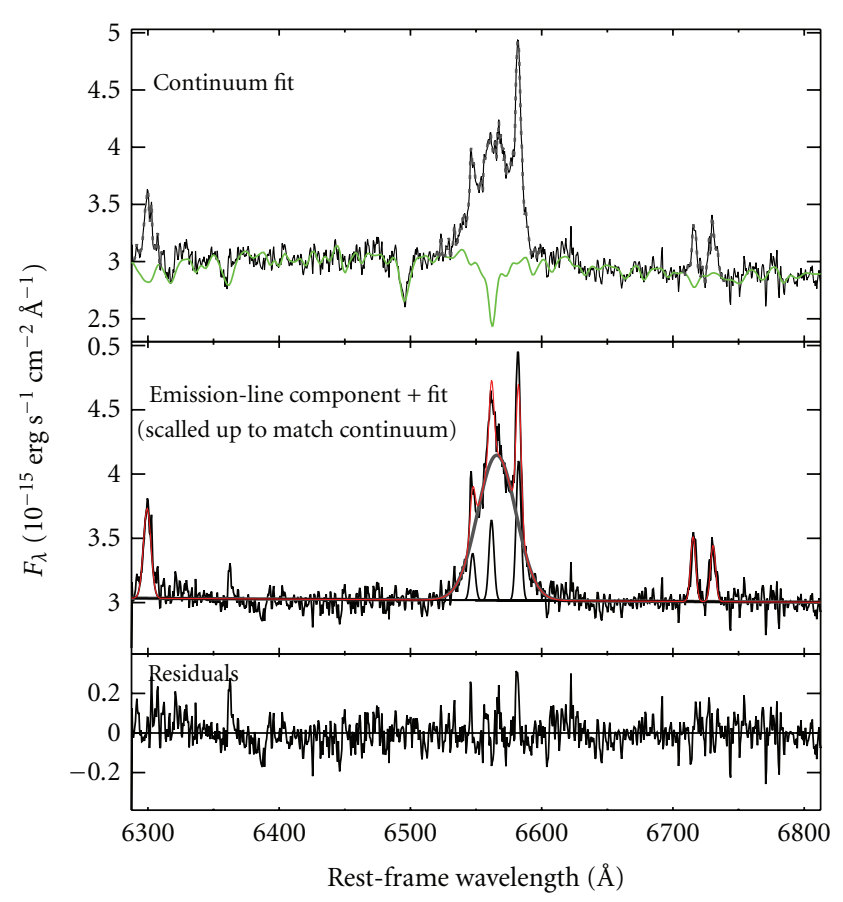

FIgure 1: Spectral fits to the HST-STIS spectrum of NGC 4736, based on a 5-pixel extraction aperture, which corresponds to a total emission area of $0.025 \mathrm{arcsec}^{2}$. Upper panel: The thin continuous line shows the observed data, while the green line shows the bestfit stellar population model obtained by clipping out the hashed emission-line region. Middle panel: The continuum subtracted spectrum, that is, the emission-line component, together with the corresponding spectral fit, that have been shifted by an additive constant to match the initial continuum level, for illustration purposes. The thin continuous lines show the individual Gaussian components (1 per narrow line), the thick grey continuous line represents the broad $\mathrm{H} \alpha$ feature, and the red line is the final fit to the observed spectrum. Lower panel: The residuals after the subtraction of the model fit.

TABle 1: Broad $\mathrm{H} \alpha$ emission measurements.

\begin{tabular}{lcl}
\hline Quantity & Value & Notes \\
\hline$f_{\text {blend }}$ & 0.80 & $\begin{array}{l}\text { Fraction of broad } \mathrm{H} \alpha \text { to } \\
\mathrm{H} \alpha+[\mathrm{N} \mathrm{II}] \text { blend. }\end{array}$ \\
$f_{\mathrm{H} \alpha}$ & 0.93 & Fraction of broad $\mathrm{H} \alpha$ to total $\mathrm{H} \alpha$ \\
$\mathrm{FWHM}\left(\mathrm{H} \alpha^{\text {broad }}\right)$ & 1570 & $\pm 110 ;$ in $\mathrm{km} \mathrm{s}^{-1}$ \\
$\Delta \mathrm{v}$ & 140 & $\pm 20 ;$ in $\mathrm{km} \mathrm{s}^{-1}$; broad relative to \\
$\log F\left(\mathrm{H} \alpha^{\text {broad }}\right)$ & -13.38 & Obrrow $\mathrm{H} \alpha$ \\
$\log L\left(\mathrm{H} \alpha^{\text {broad }}\right)$ & 37.96 & Observed flux $\left(\mathrm{erg} \mathrm{s}^{-1} \mathrm{~cm}^{-2}\right)$ \\
\hline
\end{tabular}

a assuming distance $d=4.3 \mathrm{Mpc}$.

at just $9.1 \times 10^{37} \mathrm{ergs} \mathrm{s}^{-1}$, the $\mathrm{H} \alpha$ luminosity is lower than any known broad-line sources in the Palomar sample or the Sloan Digital Sky Survey [40, 41]. This includes the famous low luminosity Seyfert 1 galaxy, NGC 4395, which has a luminosity of $1.2 \times 10^{38} \mathrm{ergs} \mathrm{s}^{-1}$ [42] and is at a similar distance to NGC 4736 . Note also that the broad $\mathrm{H} \alpha$ feature is redshifted by $\sim 140 \pm 20 \mathrm{~km} \mathrm{~s}^{-1}$ relative to the narrow 
emission, that is locked at the systemic velocity of the object; velocity offsets between the broad $\mathrm{H} \alpha$ and narrow lines are not unusual [43].

Evidence for broad $\mathrm{H} \alpha$ emission has also been presented in a recent PCA tomography study applied to this nucleus [44]. The observations reported in this case come from the Gemini Multi Object Spectrograph (GMOS)-IFU data cube and have been obtained 4 years after the HST-STIS spectrum had been acquired. Thus, the broad $\mathrm{H} \alpha$ line associated with the nucleus of M94 appears to be persistent for at least 4 years. They derive a broad $\mathrm{H} \alpha$ luminosity of $6 \times 10^{38} \mathrm{ergs} \mathrm{s}^{-1}$, $\gtrsim 6$ times brighter in the GMOS data than the one we detect in the STIS spectrum. The difference could result from true variability in the broad-line luminosity or could be due to differences in aperture size or placement. We note that Steiner et al. [44] suggest the broad-line region is offset from the photocenter of the galaxy by $0 .{ }^{\prime \prime} 15$, while our spectral $0 .{ }^{\prime \prime} 25 \times 0 .{ }^{\prime \prime} 1$ aperture coincides with the photocenter. Even with their higher $\mathrm{H} \alpha$ luminosity, this source is among the least luminous known broad-line AGN.

2.2. Ground-Based Optical Spectroscopy and the Spectral Classification. Ground-based optical spectra of the NGC 4736 nucleus are available from the Palomar spectroscopic survey by Ho et al. [43] and the integrated spectrophotometric survey of Moustakas and Kennicutt [45] conducted with the $2.3 \mathrm{~m}$ Bok telescope, as well as from new data we acquired on February 2008 at the MMT Observatory. The MMT spectrum is obtained with the Blue Chanel Spectrograph, the 500 grooves $/ \mathrm{mm}$ grating used in first order with the $1^{\prime \prime}$ slit, and covers $\lambda \lambda 3800-7000$ with $3.6 \AA$ resolution. None of these data show indications of broad $\mathrm{H} \alpha$ emission. This outcome is not surprising given the feature's flux in the HST spectrum, which would be very difficult to discern in the $1^{\prime \prime}$ or $2^{\prime \prime}$ apertures employed in these ground-based observations, which are at least one order of magnitude larger than that used in the HST-STIS observations. Through a $2^{\prime \prime}$ aperture ( 40 pc), NGC 4736's emission complex $\mathrm{H} \alpha+[\mathrm{NII}]$ is generally heavily swamped by the host stellar light.

Probably as expected, measurements of NGC 4736's narrow-line emission are consistent with this system being powered at least partially by a nonstellar source, regardless of the resolution of its observation. Figure 2 shows the location of this nucleus in the 3-dimensional diagnostic diagram usually employed in classifying emission-line galaxies (e.g., $[3,4,6])$, for different sets of data mapping $\sim 10$ to $\sim 100 \mathrm{pc}$. As with the HST-STIS observations, the ground-based measurements are performed after the host stellar contribution has been subtracted from the observed spectrum by means of absorption galaxy template fits. Our MMT spectrum offers only an upper limit for the [O III ]/H $\beta$ while the Palomar line flux measurements are at least $50 \%$ uncertain. We list the measurements of the nebular emission of this nucleus as observed by Palomar, Bok, MMTO, and HST-STIS in Table 2. Because measurements of the $[\mathrm{O}$ III $] / \mathrm{H} \beta$ ratio are not available in the HST spectrum, we show the highresolution measurements in Figure 2 using the $[\mathrm{O}$ III $] / \mathrm{H} \beta$ from the Palomar catalog; it is readily apparent that the highresolution data are consistent with a LINER or a Seyfert
TABle 2: Emission line measurements ${ }^{\mathrm{a}}$.

\begin{tabular}{lcccc}
\hline line name & HST-STIS & Palomar $^{\mathrm{b}}$ & Bok $^{\mathrm{c}}$ & MMTO $^{\mathrm{d}}$ \\
\hline [O I] $\lambda 6300$ & $5.03 \pm 0.31$ & 6.03 & $2.8 \pm 0.9$ & $7.0 \pm 1.0$ \\
$\mathrm{H} \alpha$ (narrow) & $3.00 \pm 0.32$ & $>25.12$ & $13.5 \pm 1.9$ & $22.0 \pm 2.4$ \\
{$[\mathrm{~N} \mathrm{II}] \lambda 6583$} & $5.25 \pm 0.25$ & 54.01 & $21.5 \pm 1.5$ & $39.3 \pm 2.9$ \\
[S II] $\lambda 6716$ & $2.48 \pm 0.18$ & 18.59 & $8.3 \pm 1.1$ & $15.5 \pm 0.3$ \\
[S II] $\lambda 6731$ & $2.16 \pm 0.18$ & 16.33 & $7.2 \pm 1.1$ & $9.8 \pm 0.3$ \\
\hline
\end{tabular}

aAll fluxes are in units of $10^{-15} \mathrm{erg} \mathrm{s}^{-1} \mathrm{~cm}^{-2}$ and represent the observed values, not corrected for reddening.

${ }^{\mathrm{b}}$ nonphotometric conditions; line ratios are at least $50 \%$ uncertain.

c2." 5 slit, $2.3 \mathrm{~m}$ telescope; photometric conditions [45].

${ }^{\mathrm{d}} 1^{\prime \prime}$ slit; fluxes are in units of $10^{-15} \mathrm{erg} \mathrm{s}^{-1} \mathrm{~cm}^{-2}$.

classification for this object and reveal its type 1 (broadlined) character.

2.3. The Radio, UV, and X-Ray Observations and the Astrometric Coincidence with the Broad $\mathrm{H} \alpha$ Emission. Across the electromagnetic spectrum, the center of this galaxy exhibits a complex morphology. There is a plethora of bright radio, UV, and X-ray sources detected in the center of NGC 4736. There is a nuclear compact $(15 \mathrm{GHz})$ radio source measured by Nagar et al. [46] that appears to be associated with that of the brightest of the two close $(8.49 \mathrm{GHz})$ radio sources revealed by Körding et al. [31], and with that of the brightest of the two ultraviolet point sources, which varies on a 10-year time scale [29]. Chandra observations [30] reveal numerous discrete $\mathrm{X}$-ray sources in the inner galaxy; the second brightest X-ray source, X2 $\left(L_{X, 2-10 \mathrm{keV}}=5.9 \times\right.$ $10^{38} \mathrm{erg} \mathrm{s}^{-1}$ ), coincides within the errors with the nucleus position. The off-nuclear radio, UV, and X-ray sources are apparently unrelated to each other.

The relation to possible optical counterparts of these observations is not well constrained in the literature. We thus investigated this issue, with a particular interest in the degree to which our newly detected broad $\mathrm{H} \alpha$ coincides in position with the multi- $\lambda$ detections. We used for this purpose a variety of archival images from the HST: WFPC2/PC data in the F555W filter data (PID: 5741 and 10402), HRC data in the F250W and F330W filters (PID: 9454), and NICMOS/NIC3 data in the F160W filter (PID: 9360). All data were downloaded from the HST archive, and images were drizzled together when required. Absolute astrometry was performed on these data as well as the STIS observations (taken with the slit out) using a ground-based V-band image obtained from the Spitzer Infrared Nearby Galaxies Survey (SINGS) ancillary data by Kennicutt et al. [48]. These SINGS observations were aligned to the USNO-B system using $\sim 80$ stars, and then astrometry of the HST data was obtained by degrading the resolution of the F555W image to match the SINGS image. All other HST images were then matched with the astrometry-corrected F555W frame to an accuracy of $<0$." 05 . The absolute error on this astrometry is about $0 . " 2$ and is dominated by scatter of stars in the SINGS image relative to the USNO-B positions.

Figure 3 illustrates the result of this data compilation and the corresponding radio, $\mathrm{UV}$, and X-ray source matches. It is 


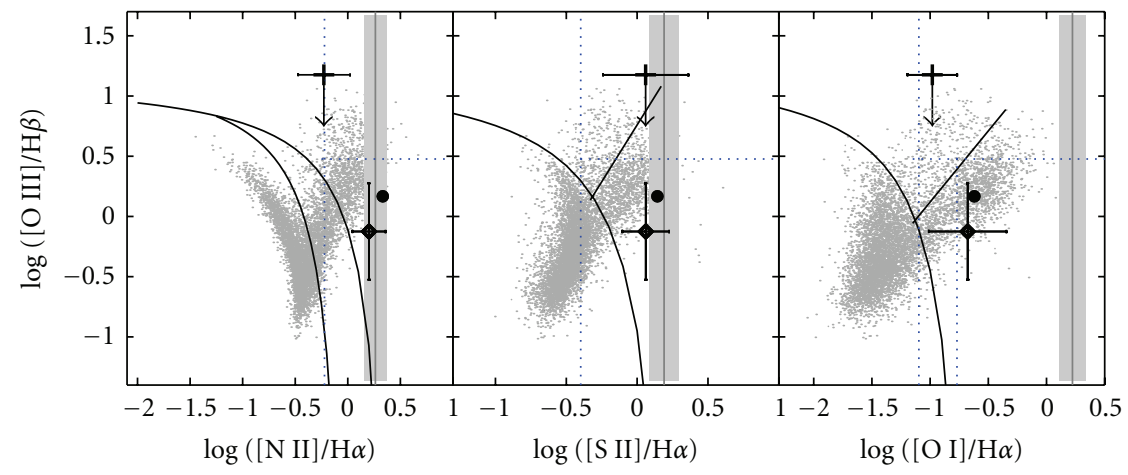

FIGURE 2: NGC 4736 within emission-line diagnostic diagrams. Filled circles reflect measurements based on the Palomar observations. Diamonds represent measurements from Moustakas and Kennicutt Jr. [45], while crosses show measurements from our MMT spectrum. The vertical bands indicate the HST-STIS measurements and the associated errors (with no correction for reddening; [O III] and H $\beta$ are not available in the HST spectra). The solid (black) curves indicate the Kewley et al. [6] classification, while the dotted (blue) lines indicate criteria used by Ho et al. [43]. The background grey points correspond to measurements of SDSS nearby galaxies from Constantin et al. [19].

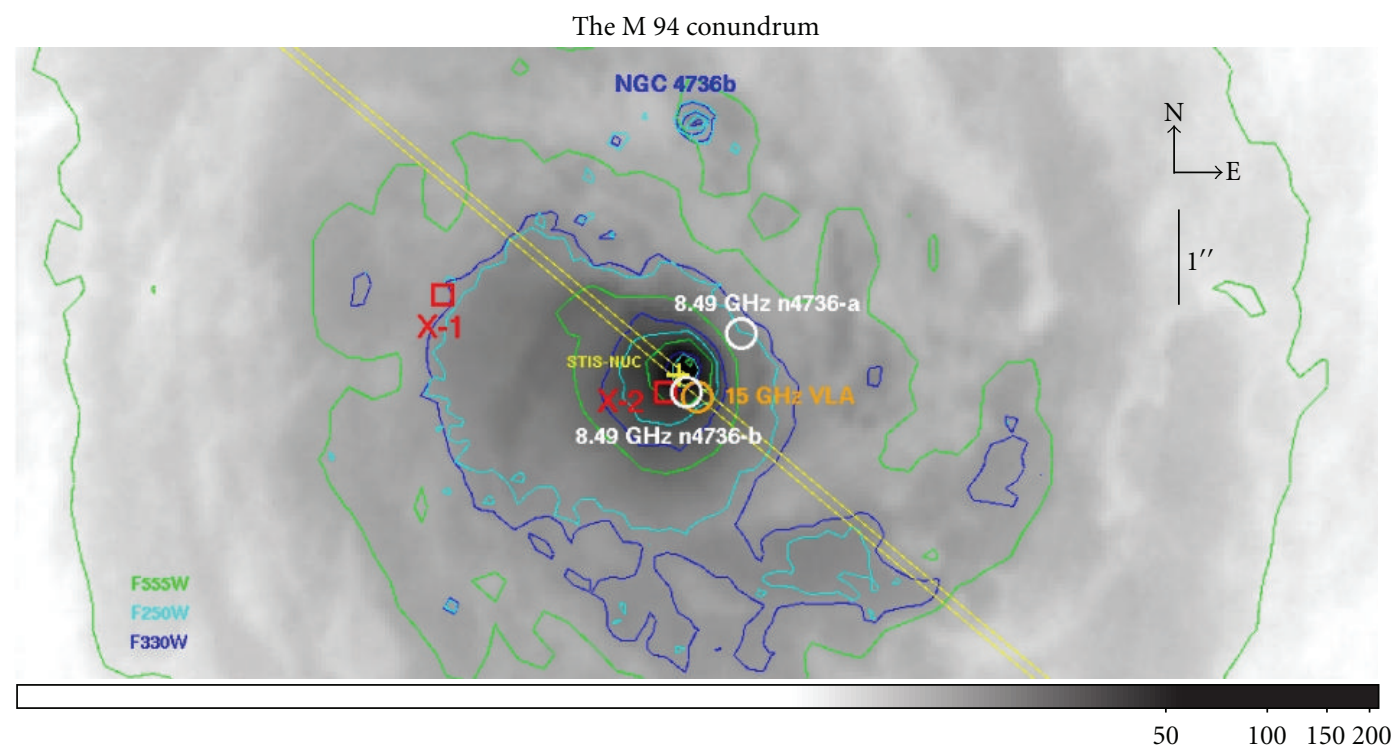

Figure 3: The mosaic-ed F555W (V-band; combination of 5 WFPC2 images taken at two different epochs) image of the NGC4736 nucleus, with intensity contours overlaid (green). The position of the STIS-nucleus is indicated (by the yellow slit) along with the locations of the 2 most luminous and closest to the nucleus hard X-ray sources, X1 and X2, as detected with Chandra (red squares), along with the position of the $15 \mathrm{GHz}$ VLA radio core ([46], orange circle), and those of the two radio compact sources detected by Körding et al. [31] (white circles). The blue and cyan contours correspond to the F330W and F250W observations from Maoz et al. [29]; note the presence of NGC 4736b,

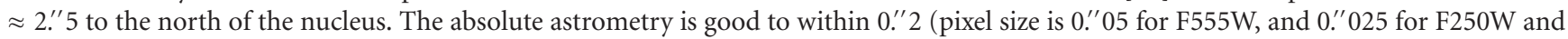
F330W).

clear that, within < 0.' 2 (4pc), there is an obvious astrometric match in the nuclear X-ray, UV, optical, radio compact sources, and the newly detected broad $\mathrm{H} \alpha$ emission line. The nucleus position as observed by STIS has RA: $12 \mathrm{~h} 50 \mathrm{~m} 53 \mathrm{s.20}$, DEC: $41^{\circ} 07^{\prime} 13$.' $^{\prime} 40$. The off-nuclear X1, that is the brightest compact X-ray source detected in this galaxy nucleus, has no counterpart at other wavelengths. Same is true for the offnuclear $8.49 \mathrm{GHz}$ detection, that is only $1^{\prime \prime}(\sim 20 \mathrm{pc})$ away from the nucleus. The off-nuclear UV source is the only one detected in optical light.

\section{NGC 4736's Nuclear Emission across the Electromagnetic Spectrum and $L_{\mathrm{Bol}}$}

With the wealth of data available for this galaxy nucleus, we are able to build its least contaminated X-ray to radio nuclear spectral energy distribution (SED). The multiwavelength observations of the sources detected in the very central regions of NGC 4736, plotted in $\nu L v$ units, are displayed in Figure 4. The X-ray detection X2 is represented here by a power law, estimated based on its photon indices $\Gamma=1.6$, 


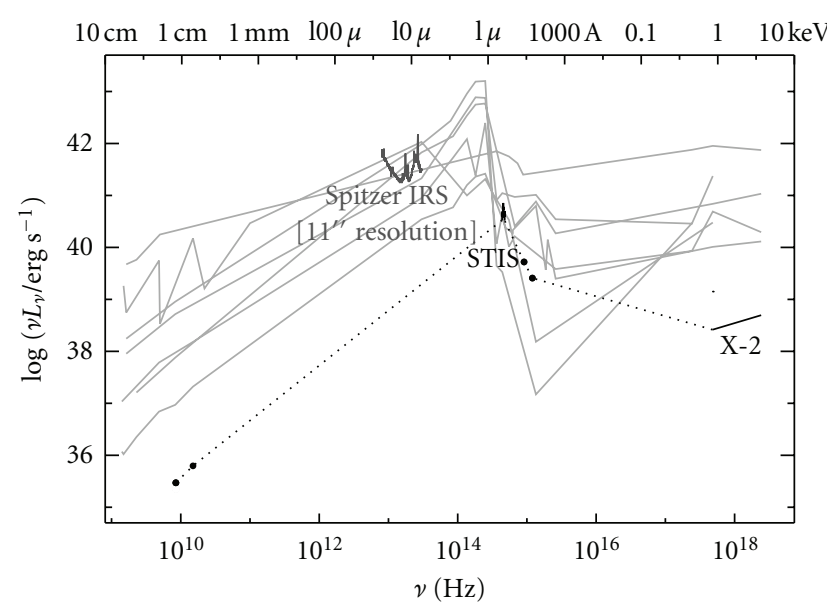

Figure 4: Radio to X-ray nuclear SED of NGC 4736 superposed on SEDs of LLAGN from Ho [47]. The optical STIS spectrum stands out as an amorphous blob featuring the strong $\mathrm{H} \alpha$ emission feature. The $2-10 \mathrm{keV}$ Chandra X-ray detection X2 is depicted as a powerlaw corresponding to $\Gamma=1.6$. The low spatial resolution Spitzer IRS observations are shown for comparison.

where the absorbing column density is fixed at the Galactic value. Both F250W and F330W UV observations are plotted, only for the nuclear detections. The optical data are represented by the HST-STIS spectrum, featuring the strong $\mathrm{H} \alpha$ emission line. Measurements corresponding to all the radio observations discussed above are indicated. For the sake of completeness, we also include in this plot lower spatialresolution observations from SINGS IRS [48] as well. We show all of these measurements superposed on the SEDs of LLAGN from Ho [47]. No artificial normalization has been performed, and there is no correction for absorption in either the Galactic extinction (except for the X-ray data) or intrinsic to NGC 4736.

With X2 and n4736-b as the X-ray and the $8.5 \mathrm{GHz}$ counterparts of the broad $\mathrm{H} \alpha$ detection, respectively and assuming that the continuum could be described as simple power laws between the points we present data for, this object's nuclear SED corresponds to $L_{\mathrm{bol}} \approx 2.5 \times 10^{40} \mathrm{erg} \mathrm{s}^{-1}$. Spitzer data is not included in estimating $L_{\text {bol }}$ because they do not reflect the IR emission of the uncontaminated nucleus; the aperture used in these observations is very large (see Table 3), including the entire field of view shown in Figure 3. This luminosity makes NGC 4736 one of the least luminous LINERs with strong evidence of $\mathrm{BH}$ accretion. This source is thus a critical signpost of $\mathrm{BH}$ accretion at extremely low levels. Note that this object's weak emission is most likely not caused by obscuration; Ho et al. [43] list a Balmer decrement of $\mathrm{H} \alpha / \mathrm{H} \beta=3.1$ (albeit highly uncertain, with a probable error of $\pm 100 \%$ ), and Eracleous et al. [30] provide an upper limit for the neutral hydrogen column density of $\mathrm{N}_{H}<3.3 \times$ $10^{20} \mathrm{~cm}^{-2}$.

3.1. Comparison with Other Observed LLAGN. Figure 4 shows that NGC 4736's SED is very similar to those of other LLAGN [47]. This suggests that, in spite of the significant
TABLE 3: Nuclear SED data.

\begin{tabular}{lcccc}
\hline $\log (\nu / \mathrm{Hz}) \log \left(\nu L_{\nu} / \mathrm{erg} / \mathrm{s}\right)$ & Resolution & Obs. date & Instr. \\
\hline 18.38 & 38.69 & $0 .^{\prime \prime} 15$ & $5 / 2000$ & Chandra-ACIS \\
17.68 & 38.42 & 0 ' $^{\prime \prime}$ & $5 / 2000$ & Chandra-ACIS \\
15.08 & 39.41 & $0 .{ }^{\prime \prime} 5$ & $6 / 2003$ & ACS F250W \\
14.96 & 39.72 & $0 .{ }^{\prime \prime} 5$ & $6 / 2003$ & ACS F330W \\
14.68 & 39.91 & $0 .^{\prime \prime} 1$ & $6 / 2002$ & HST-STIS \\
14.64 & 39.93 & $0 .^{\prime \prime} 1$ & $6 / 2002$ & HST-STIS \\
13.48 & 40.58 & $4 .^{\prime \prime} 7$ & $5 / 2004$ & IRS-SH \\
13.19 & 40.33 & $11 .^{\prime \prime} 1$ & $5 / 2004$ & IRS-SH/LH \\
12.91 & 40.87 & $11 .^{\prime \prime} 1$ & $5 / 2004$ & IRS-LH \\
10.18 & 35.79 & $0 .^{\prime \prime} 15$ & $1 / 2001$ & VLA, A config. \\
9.93 & 35.47 & $0 .^{\prime \prime} 24$ & $6-10 / 2003$ & VLA, A config. \\
\hline
\end{tabular}

difference in $L_{\mathrm{bol}}$, there is no fundamental transition in the accretion mode in this source compared to other LLAGN.

The similarity of the SED means that previously proposed bolometric corrections for LLAGN appear to work quite well for NGC 4736. The value estimated based on the correction to the [O III] line luminosity that Heckman et al. [49] proposed to work well for Seyferts is $L_{\text {bol }} \approx 3500 \times$ $L_{[\mathrm{O} \text { iii }]}=4.5 \times 10^{40} \mathrm{erg} \mathrm{s}^{-1}$ (here $L_{[\mathrm{O} \text { III }]}$ is measured from the ground within the Palomar survey and is not corrected for intrinsic reddening). If the more recent assessment of the $L_{\mathrm{bol}}=600 \times L_{[\mathrm{O} \text { III] }}$ bolometric correction of kauffmann and Heckman [50] is used, with $L_{[\mathrm{O} \text { III] }}$ corrected for reddening based on the Balmer decrement listed above and a $\tau \propto$ $\lambda^{-0.7}$ attenuation law [51], then $L_{\mathrm{bol}}=0.93 \times 10^{40} \mathrm{erg} \mathrm{s}^{-1}$, which compares satisfactorily with the measured $L_{\text {bol }}$. The average bolometric correction to the observed $2-10 \mathrm{keV} X-$ ray luminosity proposed by $\mathrm{Ho}_{0}$ [2], with $L / L_{X}=16$, results in a somewhat lower value, $0.94 \times 10^{40} \mathrm{erg} \mathrm{s}^{-1}$; with the more recent $L / L_{X}=50$ average bolometric correction to the $2-10 \mathrm{keV}$ luminosity of Eracleous et al. [52], $L_{\text {bol }}=$ $1.8 \times 10^{40} \mathrm{erg} \mathrm{s}^{-1}$ and, thus, very close to our integrated value. Within a typical uncertainty of $\sim 50 \%$, all of these estimates are consistent with the measured $L_{\mathrm{bol}}$ value. Other, more uncertain bolometric indicators are also consistent with our integration: the correlation between the mid-IR $[\mathrm{Ne} \mathrm{V}]$ emission-line luminosity and bolometric luminosity derived by Satyapal et al. [53] for a small sample of much brighter nearby AGN, that has a large scatter $(\sim 1$ order of magnitude), gives a $L_{\mathrm{bol}}=1.6 \times 10^{40} \mathrm{erg} \mathrm{s}^{-1}$, which is very close to our measured value.

This good match among quite a variety of bolometric estimators derived independently from the multiwavelength properties of AGN supports the AGN interpretation for this system. However, there are two details of its emission spectrum which are unlike typical broad-lined LLAGN.

First, despite the fact that the broad $\mathrm{H} \alpha$ emission of NGC 4736 is one of the weakest measured among type 1 AGN, the total $\mathrm{H} \alpha$ emission is peculiarly strong relative to the $\mathrm{X}$-ray counterpart. The ratio $L_{X}(2-10 \mathrm{keV}) / L_{\mathrm{H} \alpha}$ is $\sim 6$, and thus lower than the median of $\sim 15$ exhibited by the type 1 AGN (and low- $z$ quasars) included in, for example, Ho et al. [54] study that revealed a relatively tight correlation 
between the two types of emission. With the higher broad $\mathrm{H} \alpha$ flux from Steiner et al. [44], the ratio is even lower, $L_{X}(2-10 \mathrm{keV}) / L_{\mathrm{H} \alpha} \approx 1$. This finding is surprising given that the low-luminosity nearby galaxies that deviate from the $L_{X}-L_{\mathrm{H} \alpha}$ linear scaling toward lower values of the $L_{X} / L_{\mathrm{H} \alpha}$ ratios are the type 2 sources, mostly transition objects, where the ionization mechanism is not necessarily dominated by an AGN type of source. Following, for example, Ho et al. [54] arguments, the unusually low $L_{X} / L_{\mathrm{H} \alpha}$ ratio measured in the nucleus of NGC 4736 suggests that either: (1) the optical line emission is not powered exclusively by a central AGN, or (2) the X-ray emission in this system arises, at least partially, from a non-AGN source, for example, an X-ray binary. A final possibility is that the source is highly variable (as suggested by the UV, X-ray, and $\mathrm{H} \alpha$ observations), and thus, the unusual $L_{X} / L_{\mathrm{H} \alpha}$ is the result of measurements made at different times.

Second, NGC 4736's nebular emission shows a number of peculiarities. To start with, the electron density in NGC 4736's nebular emission is very low, and it does not show the typical gradient exhibited by AGN, that is, increase toward the more nuclear regions (Constantin et al. 2012, in prep.). In both the Palomar and HST-STIS observations, the ratio [S II] $\lambda \lambda 6716 / 6731$ is $\sim 1.26$ (Table 2), implying an electron density of $n_{e} \sim 10^{2} \mathrm{~cm}^{-3}$, which lies at the lowest end in the distribution of particle densities measured in the Palomar objects [55].

Given the lack of a density gradient in the line-emitting region it is then rather peculiar to observe a significant increase (by a factor of $\sim 6$ ) in the $[\mathrm{OI}] / \mathrm{H} \alpha$ line flux ratio in the HST spectrum relative to the large aperture observations, which would usually be interpreted as an indication for a more pronounced AGN-like ionization in the more central regions. There are two reasons for this: (1) [OI] requires a significantly hard radiation field, that is, that of an AGN, to sustain a sufficiently extensive partially ionized zone in clouds optically thick to Lyman continuum and thus to produce such a strong feature. Since the ionization potential of $[\mathrm{O} \mathrm{I}]$ matches that of $\mathrm{H}$ very well, large differences in the $[\mathrm{O} \mathrm{I}] / \mathrm{H} \alpha$ ratios are expected between accretion and nonaccretion sources. (2) given the lack of a density stratification in this nucleus, the degree of ionization of the emitting gas is expected to diminish with radius as the ionizing radiation emerging from a nuclear source falls off in density as $r^{-2}$ and thus produce strong gradients in the $[\mathrm{OI}] / \mathrm{H} \alpha$ ratio.

In summary, NGC 4736 may be a broad-lined AGN, with an SED similar to other LLAGN, but it is atypical in several aspects whose physical origin remains unclear. Given the above-listed possible explanations for the peculiar $L_{X} / L_{\mathrm{H} \alpha}$ ratio, we evaluate in the next subsection whether photoionization by the weak AGN in this system is sufficiently powerful to balance the emission cooling in this system.

3.2. Comparison of Ionizing and Emission-Line Power. The multiwavelength observations of this system allow for a relatively rigorous assessment of whether the photoionization by this system's AGN can power the measured emission-line luminosities and in particular that of the broad $\mathrm{H} \alpha$ component. Following Eracleous et al. [18], we can run an energy budget test via a direct comparison of the $\mathrm{H} \alpha$ luminosity and count rate with the ionizing luminosity $L_{i}=L_{1 \mathrm{Ry}-100 \mathrm{keV}}$ and the ionizing photon rate $Q_{i}=Q_{1 \mathrm{Ry}-100 \mathrm{keV}}$.

It is important to treat separately the broad and narrow emission line features as there is strong evidence that they originate from regions of significantly different physical conditions. The broad $\mathrm{H} \alpha$ comes from a much more compact and much denser emitting gas than the narrow Balmer and forbidden lines. The difference in density is at least 3 orders of magnitude; the critical density for collisional excitation of the $[\mathrm{OI}]\left(\sim 2 \times 10^{6} \mathrm{~cm}^{-3}\right)$ can be used to estimate the gas density of the broad-line region, as [O I] does not exhibit a broad component in this nucleus' emission. The difference in size is expected to be $\sim 2$ orders of magnitude (e.g., reverberation mapping of nearby Seyfert galaxies measured to be $<1$ week, [56]). Thus, the mechanisms that can operate in these two regions are expected to be qualitatively different. The photon and energy balance conditions should reflect these differences and thus should differ as well; in particular, potential contributions to the $\mathrm{H} \alpha$ emission via collisional excitation should be minimal for the narrow-line-emitting region, but important for the broad component.

Based on photoionization models (i.e., Cloudy, v94.0; see [57]) computed by Lewis et al. [58] for a wide range of ionization parameters, densities, and metallicities, Eracleous et al. [18] find that energy balance in a line-emitting nebula requires that $L_{i}>18( \pm 2) L_{\mathrm{H} \alpha} / f_{c}$, where $f_{c}$ is the covering factor or the fraction of the ionizing luminosity of the AGN that is absorbed by the line-emitting gas. These models are covering electron densities that appear to encompass both the broad- and the narrow-line-emitting regions in NGC 4736; thus, with only a fraction of $10 \%$ of ionizing photons being absorbed by the line-emitting gas, the minimum energy balance condition for AGN ionization is given by $L_{i} / L_{\mathrm{H} \alpha}>180$, for both narrow and broad emission features. In the same time, however, a minimum requirement for photon balance can be quite different for the two emission regions: for the narrow-line component $Q_{i}>2.2 Q_{\mathrm{H} \alpha}$, corresponding to the case $\mathrm{B}$ recombination (i.e., one $\mathrm{H} \alpha$ photon is emitted for every 2.2 recombinations); for the denser broad-line-emitting region, the number of $\mathrm{H}$-alpha photons that can be produced for each ionization can be at least 7-8 times higher than the standard case B estimate or $Q_{i} \gtrsim 0.25 Q_{\mathrm{H} \alpha}$ (e.g., [37]).

With the $L_{i}$ and $Q_{i}$ values already calculated by Eracleous et al. [18] by integrating M94's nuclear SED assuming that pairs of points could be connected by a power law, and with the $\mathrm{H} \alpha$ measurements from all HST and groundbased observations presented above, we can proceed with the comparison. Table 4 lists the $L_{\mathrm{H} \alpha}, Q_{\mathrm{H} \alpha}$, and associated ratios $L_{i} / L_{\mathrm{H} \alpha}, Q_{i} / Q_{\mathrm{H} \alpha}$ for all of these optical spectroscopic observations, where the narrow and broad $\mathrm{H} \alpha$ measurements are shown separately.

It is readily apparent that while $L_{i} / L_{\mathrm{H} \alpha}$ is in general $>18$ $\left(f_{c}=1\right)$, it is almost never $>180\left(f_{c}=0.1\right)$; the only exception is the narrow $\mathrm{H} \alpha$ emission measured in the HST aperture. Thus, a dominant AGN ionization of the narrow-line 
TABle 4: Ionizing and emission-line power.

\begin{tabular}{lcccc}
\hline & $\log L_{\mathrm{H} \alpha}{ }^{\mathrm{a}}$ & $\log Q_{\mathrm{H} \alpha}{ }^{\mathrm{a}}$ & $L_{i}=L_{\mathrm{H} \alpha}{ }^{\mathrm{b}}$ & $Q_{i}=Q_{\mathrm{H} \alpha}{ }^{\mathrm{c}}$ \\
\hline Palomar & $>37.7$ & $>49.3$ & $<93$ & $<2.75$ \\
Bok & 37.5 & 49.0 & 165.9 & 4.9 \\
MMTO & 37.5 & 49.2 & 104.7 & 3.1 \\
HST-STIS, narrow & 36.8 & 48.4 & 759 & 22.4 \\
HST-STIS, broad & 37.9 & 49.5 & 57.5 & 1.6 \\
PCA tomography & 38.8 & 50.3 & 8.7 & 0.25 \\
\hline
\end{tabular}

a the luminosities are measured in $\mathrm{erg} \mathrm{s}^{-1}$ and the photon rates in $\mathrm{s}^{-1}$.

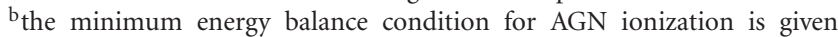
by $L_{i} / L_{\mathrm{H} \alpha} \gtrsim 180$ (when a fraction of $10 \%$ of ionizing photons photons is absorbed by the line-emitting gas).

${ }^{c}$ the minimum photon balance condition for AGN ionization is given by $Q_{i} / Q_{\mathrm{H} \alpha}>2.2$ for the narrow line regions, and $Q_{i} / Q_{\mathrm{H} \alpha} \gtrsim 0.25$ for the broad component.

region in this nucleus is definitely possible in the HST aperture, but only for $f_{c} \gtrsim 18 \%$ at larger radial distances. For the HST broad $\mathrm{H} \alpha$ feature, an AGN ionization is possible only if $f_{c} \gtrsim 30 \%$. The PCA tomography measurement of the broad $\mathrm{H} \alpha$ argues, however, against a balanced energy budget originating entirely in an AGN-like power mechanism, even when a maximum covering factor is considered; the AGNproduced energy falls short of the required amount by at least $50 \%$. Interestingly, the measured $Q_{i} / Q_{\mathrm{H} \alpha}$ ratio is well within the required photon balance corresponding to an AGN excitation for both the narrow and the broad emission features. Thus, with one clear exception, the AGN in NGC 4736 appears to be capable of providing enough photons to explain the observed $\mathrm{H} \alpha$ luminosity but only for relatively high $f_{c}$ values.

Simply because the AGN-like SED of NGC 4736 can explain the majority of the ionization energy and photon rate does not imply that the actual mechanism is an AGN, alternative excitation mechanisms must be explored. Possible options are (i) we are missing ionizing photons from accretion onto the central $\mathrm{BH}$, or (ii) there are other power sources that could make up the power deficit in this system, particularly for producing the broad $\mathrm{H} \alpha$ feature.

The first alternative could be possible if we were observing an "echo" of a previous epoch of more violent accretion, a few hundred years ago. This idea has been explored by Eracleous et al. [59] who showed that the reverberation of an ionizing flare in the nebula can produce LINER-like emission-line ratios. In this scenario, it is expected that the central UV source and the [O III] line would also follow the decay of the ionizing continuum; while the UV observations are not providing clear evidence for such a decay over the course of one decade, the multiple optical spectroscopic observations that we present in Figure 2 are consistent with a possible decrease in time (a few years) in the [O III] flux (in this model, $\mathrm{H} \beta$, [S II], [O I] are expected to decay very slowly, in $60-250$ years). Nevertheless, this duty-cycle hypothesis also requires that the broad-line emission fades immediately if the ionizing continuum declines, and the PCA tomography observations show that this is not happening in this source. The echo of such an ionizing continuum flare should also be detectable in a narrow-band [O III ] $\lambda 5007$ image in the form of a ring, which is not readily observed in M94.

The second alternative of power sources other than accretion has been often proposed in explaining the emissionline spectra of LINERs, with mixed success (see Section 1). The most probable alternative sources appear to be the mechanical power delivered by compact radio jets, along with photoionization by young or post-AGB stars from old or intermediate-age stellar populations. Shock models are highly unlikely to produce broad emission features and, thus, are not favored in this case. Recent star-formation activity remains, however, a viable option, particularly in light of relatively new discoveries of peculiar supernovae with broad $\mathrm{H} \alpha$ features that do not appear to fade in time. This idea is discussed in more detail in Section 5.

\section{The Black Hole Mass}

Given the unusual energetics of M94's nucleus, it is important to investigate whether the $\mathrm{BH}$ mass estimators derived for rapidly accreting Seyferts, which appear to be widely used for AGN, also work in the low-luminosity regime flagged by this particular system.

There are a variety of indirect methods that can be used to estimate the mass of $\mathrm{BHs}$ in galaxy centers. The available multiwavelength measurements of the nuclear emission for NGC 4736 allow the calculation of $M_{\mathrm{BH}}$ based on four different techniques, as well as with the $M-\sigma^{*}$ relation. In this section, we explore and compare the results of these five methods, along with their consequences for this object's energetics, and a comparison with a recent dynamical measurement of 6.68(5.14-8.22) $\times 10^{6} M_{\odot}$ for this object, which is listed in Kormendy et al. [60] as obtained from (Gebhardt et al. 2012, in prep.). We first present the methods and associated $M_{\mathrm{BH}}$ calculations and then discuss the shortcomings of each measurement.

(1) Using the $M-\sigma^{*}$ relation, established for quiescent nearby galaxies, including both ellipticals and spirals with classical bulges, as quantified by Gültekin et al. [1], for $\sigma^{*}=$ $110 \pm 5 \mathrm{~km} \mathrm{~s}^{-1}[61], M_{\mathrm{BH}}=1.05 \pm 0.64 \times 10^{7} M_{\odot}$; within errors, this value agrees well with Gebhardt et al. dynamical measurement. With this value, $L_{\mathrm{bol}} / L_{\mathrm{Edd}} \approx 2 \times 10^{-5}$, which is consistent with the range of values within LINERs are expected to (e.g., $[2,62])$.

(2) Using the HST measurements of the FWHM and the luminosity of the broad $\mathrm{H} \alpha$ component (see Table 1) within the scaling relation based solely on observations of this broad emission feature, as derived by Greene and Ho [63], we obtain $M_{\mathrm{BH}} \approx 3 \times 10^{4} M_{\odot}$; the fractional uncertainty associated with this measurement is $\sim 30 \%$, and includes both the scatter in the scaling relation and the errors in the line measurements. With the broad $\mathrm{H} \alpha$ line luminosity measured in the PCA tomography study [44], the BH mass would increase (by a factor of 6.5) to $M_{\mathrm{BH}}=1.9 \times 10^{5} M_{\odot}$. The difference in these two values could be considered the most conservative uncertainty associated with this $\mathrm{BH}$ mass estimate. The corresponding Eddington ratios for these $\mathrm{BH}$ masses are $L_{\mathrm{bol}} / L_{\mathrm{Edd}} \approx 1-7 \times 10^{-3}$. 
(3) Mid IR detection and measurements of the $[\mathrm{NeV}]$ $(14.32 \mu \mathrm{m})$ and [O IV] $(25.89 \mu \mathrm{m})$ emission lines [64] give, via the empirical correlations between the MIR line luminosities and reverberation mapping-based $M_{\mathrm{BH}}$ values presented by Dasyra et al. [65], a black hole mass of $2.3 \pm 0.4 \times$ $10^{5} M_{\odot}$ and $1.7 \pm 0.5 \times 10^{5} M_{\odot}$, respectively. The corresponding Eddington ratio is in this case $L_{\mathrm{bol}} / L_{\mathrm{Edd}} \approx 1.0 \times 10^{-3}$. Note that the scatter adopted for these relations is only a lower limit of the real value; thus, the uncertainty may be larger.

(4) The normalized X-ray excess variance method, as described in Papadakis et al. [66], applied to Chandra observations [30] gives $M_{\mathrm{BH}}=2.5 \pm 1.7 \times 10^{5} M_{\odot}$. To be specific, we used for this calculation the excess variance $\sigma=0.06 \pm 0.04$, $L_{\mathrm{bol}}=2.5 \times 10^{40} \mathrm{erg} \mathrm{s}^{-1}$ as derived in Section 3, and $\nu_{\mathrm{lf}}=$ $1 / T=1 / 14 \mathrm{~h}^{-1}$ (where $T$ is the length of the light curve), to estimate the break frequency $\nu_{\mathrm{bf}}$ and then $M_{\mathrm{BH}}$ via equations 4 and 6, respectively, of Papadakis et al. [66].

(5) The $M_{\mathrm{BH}}$ of this system can also be obtained via the "fundamental plane of black hole activity" that relates black hole mass to the emitted compact radio $L_{R}=v L_{\nu}(5 \mathrm{GHz})$ and hard X-ray luminosities $L_{X}(2-10 \mathrm{keV})$ and spans nine orders of magnitude in black hole mass. We calculate $L_{R}=1.7 \times 10^{35} \mathrm{erg} \mathrm{s}^{-1}$ using the two $8.5 \mathrm{GHz}$ and $15 \mathrm{GHz}$ radio measurements presented in this paper (Figure 3, Table 3 ), with a flux modeled by a powerlaw $S_{\nu} \propto v^{-\alpha}$. Using the empirical fits of Merloni et al. [67] for the fundamental plane relation, $\log \left(M_{\mathrm{BH}} / M_{\odot}\right)=5.9 \pm 1.1$ for the black hole in this system; note that this measurement and its associated errors embrace all of the estimates presented above and, thus, do not provide any additional constraint to the $M_{\mathrm{BH}}$. Interestingly, the latest derivation of the fundamental plane relation [68], applied to very low nuclear galactic luminosities, provides a $\log \left(M_{\mathrm{BH}} / M_{\odot}\right)=7.2 \pm 0.4$ for this system, which remains consistent only with the $M-\sigma^{*}$ value, as it departs considerably from those provided by the relations employing AGN emission.

There is a significant inconsistency between the value given by the $M-\sigma^{*}$ relation, which is supported by Gebhardt's dynamical measurement, and those based on the AGN emission. The $M-\sigma^{*}$ relation suggests a BH mass in NGC 4736 of $1 \times 10^{7} M_{\odot}$ and a correspondingly low $L_{\text {bol }} / L_{\text {Edd }}$ of $\sim 2 \times 10^{-5}$. Three of the estimates based on the AGN emission converge to black hole masses of $\sim 10^{5} M_{\odot}$ (and $L_{\mathrm{bol}} / L_{\mathrm{Edd}} \sim 10^{-3}$ ), showing a surprisingly consistent departure of two orders of magnitude from the $M-\sigma^{*}$ estimate. The fundamental plane relation provides a $M_{\mathrm{BH}}$ value right in between these two different situations, however, with no real additional constraint, due to its associated large uncertainty. These differences are somewhat puzzling given that the $\mathrm{BH}$ mass estimates based on nuclear emission properties are all calibrated to follow the $M-\sigma^{*}$ for high-mass black holes $\left(M_{\mathrm{BH}} \gtrsim 10^{6} M_{\odot}\right)$. It is however true that the calibrators are biased toward nearby Seyfert galaxies with much higher Eddington ratios than that of NGC 4736. We briefly discuss in the following subsections more specific weaknesses of each measurement.

4.1. Caveats of the $M-\sigma^{*}$ Relation. The $M-\sigma^{*}$ relation is expected to provide a reliable estimate of the $\mathrm{BH}$ mass as it is based on the strong correlation between dynamical mass measurements of supermassive BHs and their host properties $[1,69-71]$. This relationship is derived primarily from ellipticals and spirals with classical bulges (formed during major mergers). Recent observations suggest it may not be valid for samples of later-type spirals which more commonly host pseudobulges (formed via secular disk processes) (e.g., [72]). Because the distinction between classical and pseudobulges is based on formation, it does not simply correlate with observable properties [73, 74]. In general pseudobulges are less luminous, have lower bulge-to-total ratios, and have ongoing star formation and lower Sérsic indices than classical bulges. The possibility that the NGC 4736 is in fact a pseudobulge provides a solution to the apparent conflict in BH mass estimates.

Nevertheless, pseudobulges are difficult to identify, and there is not yet a consensus as to what defines them. To complicate things further, classical and pseudobulges can exist within the same galaxy (e.g., NGC 2787; [75]). The classification of the NGC 4736 bulge is ambiguous: Fisher and Drory [74] classifies it as a pseudobulge based on its nuclear spiral and bar and low Sérsic index $(n=1.3)$. However, they also find it has a low star formation rate, more typical of classical bulges, and thus classify it as an "Inactive Pseudobulge;" if NGC 4736 hosts a pseudobulge, it is an atypical one. We have created a surface brightness profile from NICMOS and 2MASS H-band data from the Large Galaxy Atlas [76] and found results that conflict with the fits of Fisher and Drory [74]. Specifically, we find Sérsic indices for the bulge of $n=2.3-3.0$ depending on the radial range and type of fit (single versus double Sérsic), which are consistent with a classical one (as shown in [74]).

Our surface brightness profile fits also reveal the presence of a nuclear star cluster within the central $\sim 0.6^{\prime \prime}(\sim 12 \mathrm{pc})$, with an $\mathrm{H}$-band magnitude of $\sim 12.5$. Such nuclear star clusters are common in early-type spiral galaxies [77]. The luminosity and mass of nuclear star clusters are known to scale with bulge luminosity and mass [78-80], and the NGC 4736 nuclear star cluster has a luminosity that is $0.1 \%$ of its bulge, typical for nuclear star clusters [81]. Nuclear star clusters commonly coexist with black holes, but there are a very limited number of cases where masses for both can be estimated [82]. In these cases, including the Milky Way, the $\mathrm{BH}$ mass is similar to the mass of the nuclear star cluster within an order of magnitude. There is also some evidence that the ratio of $\mathrm{BH}$ mass to nuclear star cluster mass increases with spheroid mass [83]. For NGC 4736, assuming an old population with an $\mathrm{H}$-band $M / L \sim 0.7$, the nuclear cluster would have a mass of $\sim 2 \times 10^{7} M_{\odot}$. This mass is quite similar to the $M-\sigma^{*} \mathrm{BH}$ mass estimate, suggesting thus the presence of a similar-sized $\mathrm{BH}$.

4.2. Caveats of the BLR Scaling Relation. The BLR scaling relations were derived using high-luminosity systems (i.e., Seyferts, not LINERs) and have been scaled to match the dynamical black hole detections of BHs with masses $>10^{6} M_{\odot}$. Thus, their applicability to NGC 4736 , where they yield an estimate of $\sim 10^{5} M_{\odot}$, is a (perhaps unwise) extrapolation. These relations assume that the BLR is virialized due 
to proximity to the $\mathrm{BH}[84]$ and thus the $5100 \AA$ continuum luminosity correlates with the emissivity-weighted radius of the BLR and thus with the BH mass [85]. The overall errors associated with $\mathrm{BH}$ mass measurements based on these relations do not exceed $0.5 \mathrm{dex}$ [86-88], and there appears to be good consistency with $\mathrm{BH}$ masses obtained via the $M-\sigma^{*}$ relation for relatively bright AGN $\left(L>10^{42} \mathrm{erg} \mathrm{s}^{-1}\right)$, with $M_{\mathrm{BH}} \gtrsim 10^{6} M_{\odot}$ [89]. More recent calibrations of the radius-luminosity relationship on which these techniques are based infer that $\mathrm{BH}$ masses have been overestimated, however, only by up to a factor of $\sim 3$ [90]. This latter study also indicates a trend toward larger uncertainties and larger amount of overestimation in the $\mathrm{BH}$ with decreasing luminosity; however, no conclusive results are yet available for this regime.

4.3. Caveats of the MIR Line Correlation. The Dasyra et al. [65] empirical relation between the MIR line emission properties and the $\mathrm{BH}$ mass is derived using reverberation mapping $\mathrm{BH}$ masses and, thus, as with the previous method, may not apply to systems with $M_{\mathrm{BH}}<10^{6} M_{\odot}$. The relation holds for systems with $L_{\mathrm{bol}} / L_{\mathrm{Edd}}>0.003$, but not necessarily beyond this range. The $M_{\mathrm{BH}} \approx 10^{5} M_{\odot}$ given by this method places this object at the low end of this $L_{\mathrm{bol}} / L_{\mathrm{Edd}}$ range; on the other hand, a more massive and thus quiescent $\mathrm{BH}$ would correspond to a $[\mathrm{NeV}]$ luminosity of a few orders of magnitude lower than that measured. The scatter associated with this relation remains in average $0.5 \mathrm{dex}$ and thus cannot account for the $\sim 2-3$ orders of magnitude difference between this value and that obtained via $M-\sigma^{*}$.

4.4. Issues with Estimating the BH Mass from X-Rays. The BH mass derivation based on X-ray variability, or more precisely on the relation between the excess variance and $M_{\mathrm{BH}}$, relies on the hypothesis of a universal power spectral density function (PSD) shape and amplitude in AGN, which is based on the idea that the X-ray variability mechanism and the accretion efficiency are the same for all AGN, at all redshifts. These assumptions appear to hold for the objects involved in deriving and testing this relation [66]; that sample is, however, small and rather biased toward luminous (X-ray) sources, with $\log L_{X} /\left(\mathrm{erg} \mathrm{s}^{-1}\right)>41.5$. None of the 2-10 keV sources detected in the nucleus of NGC 4736 are in this luminosity range. Nevertheless, Galactic BHs $\left(M_{\mathrm{bh}}<\right.$ $10^{2} M_{\odot}$ ) in their hard states show variability properties that match well those of AGN, both of these types of sources falling on the same projection of the $T_{B}-M_{\mathrm{bh}}-L_{\mathrm{bol}} / L_{\mathrm{Edd}}$ plane, where $T_{B}=1 / \nu_{\mathrm{bf}}$, suggesting that an extrapolation to the intermediate mass $\mathrm{BH}$ (or lower $L$ ) level is practicable.

4.5. Problems with the Fundamental Plane. Finally, it appears that the main problem with the fundamental plane is that the relation is not sharpened enough to provide strong constraints on $\mathrm{BH}$ masses. Because the relation spans nine orders of magnitude in $\mathrm{BH}$ mass, it is expected to equally apply to any value of $M_{\mathrm{bh}}$ in the range we are interested in. It is also the case that a wide variety of $\mathrm{BH}$ accretion models (e.g., with efficient and inefficient flows for the X-ray emission or associating X-ray flux with synchrotron emission near the base of a jet) are consistent with this relation (e.g., $[91,92])$, suggesting that a large diversity of accretion modes or rates are accommodated.

Nevertheless, the fundamental plane remains best constrained only for systems with $M_{\mathrm{BH}} \gtrsim 10^{6} M_{\odot}$ and for very low nuclear luminosities, that is, with negligible or zero AGN contribution that allow a dynamical measurement of their $\mathrm{BH}$ mass [68]. In this regime, the latest derivation of the fundamental plane relation provides for M94 a $M_{\mathrm{BH}}$ value consistent with the $M-\sigma^{*}$ estimate and the dynamical measurement, while it would not reliably constrain a $M_{\mathrm{BH}} \lesssim$ $10^{6} M_{\odot}$ value. The scatter in the fundamental plane relation increases for lower BH masses, with higher Eddington ratios $[68,93]$.

\section{Discussion: Alternative Power Generation Mechanisms}

The detection of broad $\mathrm{H} \alpha$ emission, combined with the spatial coincidence of this emission with the detection of $\mathrm{X}$-ray, UV, and radio compact sources, provides strong evidence that this galaxy hosts an accreting massive black hole. The SED of this nucleus makes this object one of the lowest luminosity LINER with a distinct contribution to the total emission by black hole accretion. In this scenario, the nucleus is an AGN, and the presence of off-nuclear sources, particularly the radio and the UV detections, may result from remnant jet activity emerging from the nucleus. It is definitely exciting to detect AGN activity at energy levels equal to that of several young supernova remnants of the Cas A variety [94], an OB association which hosts a high-mass Xray binary [30] or simply a group of five late $\mathrm{O}$ supergiants [28].

Nevertheless, the presence of a number of unusual offnuclear sources, coupled with the apparent deficit in the photoionizing photons, and with the fact that the $\mathrm{BH}$ mass estimates based on AGN emission appear to fail for this object, encourages us to explore alternative scenarios for the NGC 4736 nucleus. In particular, it is very likely that the $\mathrm{BH}$ mass estimates that exploit the multiwavelength AGN characteristics do not work for this system because at least some of this emission is not the result of $\mathrm{BH}$ accretion.

There are certain (peculiar) kinds of core-collapse $\mathrm{SNe}$ that present multiwavelength observations and in particular broad $\mathrm{H} \alpha$ emission components with characteristics that are very similar to those we measure in the nucleus of M94. The SN 2005ip, presented by Smith et al. [95], is a very good example. The so-called intermediate $\mathrm{H} \alpha$ component associated with this SN ejecta presents the same FWHM and brightness level as the broad $\mathrm{H} \alpha$ detected in the STIS aperture, and it does not show any sign of diminishing its strength over more than 3-year period (see their Figure 7; a very broad component with FWHM $\gtrsim 10000 \mathrm{~km} \mathrm{~s}^{-1}$ like the one exhibited by SN 2005ip would not be measurable in the galaxy spectrum as it would be completely swamped in the continuum stellar light, even in the HST-STIS observations).

$\mathrm{SNe}$ as luminous as X2, with intrinsically hard Xray spectra (photon index $\Gamma \lesssim 1$ ), have certainly been encountered, for cases observed few years after the explosion 
(e.g., ATe \#1023; [96]); for these cases, $L_{X} / L_{\text {bol }}$ ratios are high relative to more standard SN cases [97]. Another particular example of a SN that matches well the measurements of the nuclear emission in NGC 4736 is SDSS J09529.56 + 214313.3 [98], which is believed (but not confirmed) to be a SN type IIn. For this system, the broad $\mathrm{H} \alpha$ component stays strong for at least three years, and its $L_{X}(2-10 \mathrm{keV}) / L_{\mathrm{H} \alpha} \approx 1$, matching thus very well the surprisingly low value measured for NGC 4736.

The peculiar nebular, radio, and UV characteristics of the nucleus of NGC 4736 appear to also compare well the SN phenomenology. The electron densities (or [S II] line flux ratios) measured in this galaxy center are matching exactly the ones measured in the environments of the extraordinary type IIn SNe we compare here with, for example, SDSS J09529.56 + 214313.3. The two different band radio observations of this nucleus are consistent with emission from extragalactic $\mathrm{SNe}$, in both intensity and decline rate in flux density [99-101]. Moreover, the compact radio offnuclear detection, only $1^{\prime \prime}$ (20 pc) away from the nuclear one [31], could be interpreted as the result of shock emission associated with a nuclear core-collapse SN. Measurements of the brightness of the off-nuclear UV detection in F250W and $\mathrm{F} 330 \mathrm{~W}$ place this object into the $\mathrm{O}$ star of late (5-ish) type spectral category, fitting thus well into the idea that this nucleus could simply be a star-forming site and that NGC 4736a's emission includes significant contributions from a SN which exploded close to the weakly active (and massive, $\left.M_{\mathrm{BH}} \sim 10^{(6-7)} M_{\odot}\right)$ central BH.

This SN contribution scenario may have its own drawbacks. If the broad $\mathrm{H} \alpha$ emission has actually gotten brighter by a factor of 6 , as suggested by the comparison between the HST-STIS and GMOS observations, the SN interpretation becomes problematic; variability of the broad-line region originating in the AGN could, in principle, account for this effect. Also, the observed intraday (hour-scale) X-ray variability measured for $\mathrm{X} 2$ remains yet to be detected in a supernova and conflicts with the physical scale over which Xray emission is expected in $\mathrm{SN}$ remnants; thus, this particular behavior may remain strictly associated with the AGN. Additional observations would be necessary to fully confirm or rule out the SN scenario. Specifically, new high-resolution observations in the optical, UV, or X-rays would be able to confirm if there is a fading in the light curve, as expected from a SN, and would also allow accurate localization of the source. High S/N optical spectra would much better resolve the emission-line profile and a possible temporal evolution.

\section{Conclusion}

We have presented here an exhaustive multiwavelength analysis of the nuclear emission properties of NGC 4736, prompted by new measurements of a broad $\mathrm{H} \alpha$ emission component detected in its high-resolution HST-STIS optical spectrum. This broad $\mathrm{H} \alpha$ component, with a luminosity of $9 \times 10^{37} \mathrm{ergs} \mathrm{s}^{-1}$, is one of the lowest luminosity broad line known. This broad $\mathrm{H} \alpha$ is coincident with a compact bright $\mathrm{X}$-ray and radio source. Our measurements of this object's spectral energy distribution reveal a bolometric luminosity of $L_{\mathrm{bol}} \approx 2.5 \times 10^{40} \mathrm{erg} \mathrm{s}^{-1}$, that categorizes NGC 4736 as one of the least luminous LINERs with strong evidence for $\mathrm{BH}$ accretion. Our comparison of five independent $\mathrm{BH}$ mass estimates reveals a discrepancy of two orders of magnitude between the value $\sim 10^{7} M_{\odot}$ predicted by the $M-\sigma^{*}$ relation and the value $\sim 10^{5} M_{\odot}$ toward which methods based on AGN emission activity in optical, mid-IR, and X-ray, seem to converge; the fifth method is provided by the fundamental plane relation, which, however, due to its large associated uncertainties, does not offer any additional constraint to this comparison.

We conclude that this system's $\mathrm{BH}$ mass cannot be reliably estimated via standard AGN BH mass indicators because the nuclear emission in this system is not entirely tracing the accretion onto the central $\mathrm{BH}$. Our assessment of the energy budgets of the ionizing and emission-line power suggests a possible deficit in the AGN ionization and production of a broad $\mathrm{H} \alpha$ emission feature which can be made up by a peculiar kind of Type IIn SN that matches well the nuclear emission of NGC 4736 over the whole electromagnetic spectrum and supports this galaxy nucleus' general aging starburst-like appearance.

\section{Acknowledgments}

The authors thank the anonymous referee for constructive comments that helped them improved the paper. Support for this work was provided by NASA through Grant no. HSTAR-11749.01-A from the STScI, which is operated by the Association of Universities for Research in Astronomy, Inc., under NASA Contract NAS5-26555, based on observations made with the NASA/ESA Hubble Space Telescope, obtained from the data archive at the Space Telescope Science Institute. STScI is operated by the Association of Universities for Research in Astronomy, Inc., under NASA Contract NAS 526555. Some observations reported here were obtained at the MMT Observatory, a joint facility of the Smithsonian Institution and the University of Arizona.

\section{References}

[1] K. Gültekin, D. O. Richstone, K. Gebhardt et al., "the m- $\sigma$ and $\mathrm{m}-\mathrm{l}$ relations in galactic bulges, and determinations of their intrinsic scatter," Astrophysical Journal, vol. 698, no. 1, p. 198, 2009.

[2] L. C. Ho, "Nuclear activity in nearby galaxies," Annual Review of Astronomy and Astrophysics, vol. 46, pp. 475-539, 2008.

[3] J. A. Baldwin, M. M. Phillips, and R. Terlevich, "Classification parameters for the emission-line spectra of extragalactic objects," Astronomical Society of the Pacific, vol. 93, pp. 5-19, 1981.

[4] S. Veilleux and D. E. Osterbrock, "Spectral classification of emission-line galaxies," Astrophysical Journal, Supplement Series, vol. 63, pp. 295-301, 1987.

[5] G. Kauffmann, T. M. Heckman, C. Tremonti et al., "The host galaxies of active galactic nuclei," Monthly Notices of the Royal Astronomical Society, vol. 346, no. 4, pp. 1055-1077, 2003.

[6] L. J. Kewley, B. Groves, G. Kauffmann, and T. Heckman, "The host galaxies and classification of active galactic nuclei," Monthly Notices of the Royal Astronomical Society, vol. 372, no. 3, pp. 961-976, 2006. 
[7] L. C. Ho, A. V. Filippenko, and W. L. W. Sargent, "A search for "dwarf" seyfert nuclei. V. Demographics of nuclear activity in nearby galaxies," Astrophysical Journal, vol. 487, no. 2, pp. 568-578, 1997.

[8] A. Constantin and M. S. Vogeley, "The clustering of lowluminosity active galactic nuclei," Astrophysical Journal, vol. 650, no. 2 I, pp. 727-748, 2006.

[9] D. W. Weedman, L. Hao, S. J. U. Higdon et al., "Mid-infrared spectra of classical AGNs observed with the spitzer space telescope," Astrophysical Journal, vol. 633, no. 2 I, pp. 706716, 2005.

[10] L. Armus, J. Bernard-Salas, H. W. W. Spoon et al., "Detection of the buried active galactic nucleus in NGC 6240 with the infrared spectrograph on the Spitzer Space Telescope," Astrophysical Journal, vol. 640, no. 1 I, pp. 204-210, 2006.

[11] N. P. Abel and S. Satyapal, "[Ne v] Emission in optically classified starbursts," Astrophysical Journal, vol. 678, no. 2, pp. 686-692, 2008.

[12] E. Sturm, D. Lutz, A. Verma et al., "Mid-infrared line diagnostics of active galaxies: a spectroscopic AGN survey with ISO-SWS," Astronomy and Astrophysics, vol. 393, no. 3, pp. 821-841, 2002.

[13] S. Satyapal, D. Vega, R. P. Dudik, N. P. Abel, and T. Heckman, "Spitzer uncovers active galactic nuclei missed by optical surveys in seven late-type galaxies," Astrophysical Journal, vol. 677, no. 2, pp. 926-942, 2008.

[14] A. D. Goulding and D. M. Alexander, "Towards a complete census of AGN in nearby Galaxies: a large population of optically unidentified AGN," Monthly Notices of the Royal Astronomical Society, vol. 398, no. 3, pp. 1165-1193, 2009.

[15] N. M. Nagar, H. Falcke, and A. S. Wilson, "Radio sources in low-luminosity active galactic nuclei IV. Radio luminosity function, importance of jet power, and radio properties of the complete Palomar sample," Astronomy and Astrophysics, vol. 435, no. 2, pp. 521-543, 2005.

[16] W. M. Zhang, R. Soria, S. N. Zhang, D. A. Swartz, and J. Liu, "A census of X-ray nuclear activity in nearby galaxies," Astrophysical Journal, vol. 699, no. 1, pp. 281-297, 2009.

[17] L.-B. Desroches, J. E. Greene, and L. C. Ho, "X-ray properties of intermediate-mass black holes in active galaxies. II. Xray-bright accretion and possible evidence for slim disks," Astrophysical Journal, vol. 698, p. 1515, 2009.

[18] M. Eracleous, J. A. Hwang, and H. M. L. G. Flohic, "An assessment of the energy budgets of low-ionization nuclear emission regions," Astrophysical Journal, vol. 711, no. 2, pp. 796-810, 2010.

[19] A. Constantin, F. Hoyle, and M. S. Vogeley, "Active galactic nuclei in void regions," Astrophysical Journal, vol. 673, no. 2, pp. 715-729, 2008.

[20] A. Constantin, P. Green, T. Aldcroft et al., "Probing the balance of AGN and star-forming activity in the local universe with ChaMP," Astrophysical Journal, vol. 705, no. 2, pp. 1336-1355, 2009.

[21] K. Schawinski, D. Thomas, M. Sarzi et al., "Observational evidence for AGN feedback in early-type galaxies," Monthly Notices of the Royal Astronomical Society, vol. 382, no. 4, pp. 1415-1431, 2007.

[22] K. Schawinski, C. M. Urry, S. Virani et al., "Galaxy zoo: the fundamentally different co-evolution of supermassive black holes and their early- and late-type host galaxies," Astrophysical Journal, vol. 711, no. 1, pp. 284-302, 2010.

[23] T. Di Matteo, V. Springel, and L. Ilernquist, "Energy input from quasars regulates the growth and activity of black holes and their host galaxies," Nature, vol. 433, no. 7026, pp. 604607, 2005.

[24] P. F. Hopkins, L. Hernquist, T. J. Cox, T. Di Matteo, B. Robertson, and V. Springel, "A unified, merger-driven model of the origin of starbursts, quasars, the cosmic X-ray background, supermassive black holes, and galaxy spheroids," Astrophysical Journal, Supplement Series, vol. 163, no. 1, pp. $1-49,2006$.

[25] R. B. Tully and J. R. Fisher, Catalog of Nearby Galaxies, Cambridge University Press, Cambridge, UK, 1988.

[26] A. V. Filippenko and W. L. W. Sargent, "A search for "Dwarf" seyfert 1 nuclei. I. The initial data and results," Astrophysical Journal, Supplement Series, vol. 57, pp. 503-522, 1985.

[27] P. F. Spinelli, T. Storchi-Bergmann, C. H. Brandt, and D. Calzetti, "An atlas of Hubble space telescope stis spectra of Seyfert galaxies," Astrophysical Journal, Supplement Series, vol. 166, no. 2, pp. 498-504, 2006.

[28] D. Maoz, A. V. Filippenko, L. C. Ho et al., "Detection of compact ultraviolet nuclear emission in liner galaxies," Astrophysical Journal, vol. 440, no. 1, pp. 91-99, 1995.

[29] D. Maoz, N. M. Nagar, H. Falcke, and A. S. Wilson, "The murmur of the sleeping black hole: detection of nuclear ultraviolet variability in LINER galaxies," Astrophysical Journal, vol. 625, no. 2 I, pp. 699-715, 2005.

[30] M. Eracleous, J. C. Shields, G. Chartas, and E. C. Moran, "Three LINERs under the Chandra X-ray microscope," Astrophysical Journal, vol. 565, no. 1 I, pp. 108-124, 2002.

[31] E. Körding, E. Colbert, and H. Falcke, "A radio monitoring survey of ultra-luminous X-ray sources," Astronomy and Astrophysics, vol. 436, no. 2, pp. 427-436, 2005.

[32] A. L. Kinney, R. C. Bohlin, D. Calzetti, N. Panagia, and R. F. G. Wyse, "An atlas of ultraviolet spectra of star-forming galaxies," Astrophysical Journal, Supplement Series, vol. 86, no. 1, pp. 5-93, 1993.

[33] B. J. Smith, P. M. Harvey, C. Colomé, C. Y. Zhang, J. DiFrancesco, and R. W. Pogge, "Far-infrared emission from the bulges of early-type spirals: kao observations of NGC 4736 (M94) and NGC 3627 (M66)," Astrophysical Journal, vol. 425, no. 1, pp. 91-102, 1994.

[34] L. Dressel, P. Hodge, and P. Barrett, "wx2d: a PyRAF routine to resample spectral images," Instrument Science Report STIS 2007-04, 2007.

[35] G. Bruzual and S. Charlot, "Stellar population synthesis at the resolution of 2003," Monthly Notices of the Royal Astronomical Society, vol. 344, no. 4, pp. 1000-1028, 2003.

[36] C. A. Tremonti, T. M. Heckman, G. Kauffmann et al., "The origin of the mass-metallicity relation: insights from 53,000 star-forming galaxies in the sloan digital sky survey," Astrophysical Journal, vol. 613, no. 2 I, pp. 898-913, 2004.

[37] D. E. Osterbrock, Astrophysics of Gaseus Nebulae and Active Galactic Nuclei, University Science Books, 1989.

[38] G. Kriss, "Fitting models to UV and optical spectral data," in Astronomical Data Analysis Software and Systems III, D. R. Crabtree, R. J. Hanisch, and J. Barnes, Eds., vol. 61 of Astronomical Society of the Pacific Conference Series, p. 437, 1994.

[39] J. L. Walsh, A. J. Barth, and M. Sarzi, “The supermassive black hole in M84 revisited," Astrophysical Journal, vol. 721, no. 1, pp. 762-776, 2010.

[40] L. C. Ho, A. V. Filippenko, W. L. W. Sargent, and C. Y. Peng, "A search for "dwarf" seyfert nuclei. IV. Nuclei with broad $\mathrm{H} \alpha$ emission," Astrophysical Journal, Supplement Series, vol. 112, no. 2, pp. 391-414, 1997. 
[41] J. E. Greene and L. C. Ho, "A new sample of low-mass black holes in active galaxies," Astrophysical Journal Letters, vol. 670, no. 1, pp. 92-104, 2007.

[42] A. V. Filippenko and W. L. W. Sargent, "Discovery of an extremely low luminosity Seyfert 1 nucleus in the dwarf galaxy NGC 4395," The Astrophysical Journal, vol. 342, pp. 11-14, 1989.

[43] L. C. Ho, A. V. Filippenko, and W. L. W. Sargent, "A search for "dwarf" seyfert nuclei. III. Spectroscopic parameters and properties of the host galaxies," Astrophysical Journal, Supplement Series, vol. 112, no. 2, pp. 315-390, 1997.

[44] J. E. Steiner, R. B. Menezes, T. V. Ricci, and A. S. Oliveira, "PCA Tomography: how to extract information from data cubes," Monthly Notices of the Royal Astronomical Society, vol. 395, no. 1, pp. 64-75, 2009.

[45] J. Moustakas and R. C. Kennicutt Jr., "An integrated spectrophotometric survey of nearby star-forming galaxies," Astrophysical Journal, Supplement Series, vol. 164, no. 1, pp. 81-98, 2006.

[46] N. M. Nagar, H. Falcke, A. S. Wilson, and J. S. Ulvesta, "Radio sources in low-luminosity active galactic nuclei. III. "AGNs" in a distance-limited sample of "LLAGNs"," Astronomy and Astrophysics, vol. 392, no. 1, pp. 53-82, 2002.

[47] L. C. Ho, "The spectral energy distributions of lowluminosity active galactic nuclei," Astrophysical Journal, vol. 516, no. 2, pp. 672-682, 1999.

[48] R. C. Kennicutt, L. Armus, G. Bendo et al., "SINGS: the SIRTF nearby galaxies survey," Publications of the Astronomical Society of the Pacific, vol. 115, no. 810, pp. 928-952, 2003.

[49] T. M. Heckman, G. Kauffmann, J. Brinchmann, S. Charlot, C. Tremonti, and S. D. M. White, "Present-day growth of black holes and bulges: the sloan digital sky survey perspective," Astrophysical Journal, vol. 613, no. 1 I, pp. 109-118, 2004.

[50] G. Kauffmann and T. M. Heckman, "Feast and Famine: regulation of black hole growth in low-redshift galaxies," Monthly Notices of the Royal Astronomical Society, vol. 397, no. 1, pp. 135-147, 2009

[51] S. Charlot and S. M. Fall, "A simple model for the absorption of starlight by dust in galaxies," Astrophysical Journal, vol. 539, no. 2, pp. 718-731, 2000.

[52] M. Eracleous, J. A. Hwang, and H. M. L. G. Flohic, "Spectral energy distributions of weak active galactic nuclei associated with low-ionization nuclear emission regions," Astrophysical Journal, Supplement Series, vol. 187, no. 1, pp. 135-148, 2010.

[53] S. Satyapal, D. Vega, T. Heckman, B. O'Halloran, and R. Dudik, "The discovery of an active galactic nucleus in the late-type galaxy NGC 3621: spitzer spectroscopic observations," Astrophysical Journal, vol. 663, no. 1, pp. L9L12, 2007.

[54] L. C. Ho, E. D. Feigelson, L. K. Townsley et al., "Detection of nuclear X-ray sources in nearby galaxies with chandra," Astrophysical Journal, vol. 549, no. 1, pp. L51-L54, 2001.

[55] L. C. Ho, A. V. Filippenko, and W. L. W. Sargent, "A search for "dwarf" Seyfert nuclei. VI. Properties of emission-line nuclei in nearby galaxies," Astrophysical Journal, vol. 583, no. 1 I, pp. 159-177, 2003.

[56] K. D. Denney, B. M. Peterson, R. W. Pogge et al., "Reverberation mapping measurements of black hole masses in six local Seyfert galaxies," Astrophysical Journal, vol. 721, no. 1, pp. 715-737, 2010.

[57] G. J. Ferland, K. T. Korista, D. A. Verner, J. W. Ferguson, J. B. Kingdon, and E. M. Verner, "CLOUDY 90: Numerical simulation of plasmas and their spectra," Publications of the
Astronomical Society of the Pacific, vol. 110, no. 749, pp. 761778, 1998.

[58] K. T. Lewis, M. Eracleous, and R. M. Sambruna, "Emissionline diagnostics of the central engines of weak-line radio galaxies," Astrophysical Journal, vol. 593, no. 1 I, pp. 115-126, 2003.

[59] M. Eracleous, M. Livio, and L. Binette, "A duty cycle hypothesis for the central engines of LINERs," Astrophysical Journal Letters, vol. 445, p. L1, 1995.

[60] J. Kormendy, R. Bender, and M. E. Cornell, "Supermassive black holes do not correlate with galaxy disks or pseudobulges," Nature, vol. 469, no. 7330, pp. 374-376, 2011.

[61] A. J. Barth, L. C. Ho, and W. L. W. Sargent, "A study of the direct fitting method for measurement of galaxy velocity dispersions," Astronomical Journal, vol. 124, no. 5, pp. 26072614, 2002.

[62] L. C. Ho, "Black hole demography from nearby active galactic nuclei," in Coevolution of Black Holes and Galaxies, L. C. Ho, Ed., the Carnegie Observatories Astrophysics Series, p. 293, Cambridge University Press, 2004.

[63] J. E. Greene and L. C. Ho, "Estimating black hole masses in active galaxies using the $\mathrm{H} \alpha$ emission line," Astrophysical Journal, vol. 630, no. 1 I, pp. 122-129, 2005.

[64] R. P. Dudik, S. Satyapal, and D. Marcu, "A spitzer spectroscopic survey of low-ionization nuclear emission-line regions: characterization of the central source," Astrophysical Journal, vol. 691, p. 1501, 2009.

[65] K. M. Dasyra, L. C. Ho, L. Armus et al., "High-ionization mid-infrared lines as black hole mass and bolometric luminosity indicators in active galactic nuclei," Astrophysical Journal, vol. 674, no. 1, pp. L9-L12, 2008.

[66] I. E. Papadakis, E. Chatzopoulos, D. Athanasiadis, A. Markowitz, and I. Georgantopoulos, "The long-term X-ray variability properties of AGNs in the Lockman Hole region," Astronomy and Astrophysics, vol. 487, no. 2, pp. 475-483, 2008.

[67] A. Merloni, S. Heinz, and T. Di Matteo, "A fundamental plane of black hole activity," Monthly Notices of the Royal Astronomical Society, vol. 345, no. 4, pp. 1057-1076, 2003.

[68] K. Gültekin, E. M. Cackett, J. M. Miller et al., "the fundamental plane of accretion onto black holes with dynamical masses," The Astrophysical Journal, vol. 706, no. 1, p. 404, 2009.

[69] L. Ferrarese and D. Merritt, "A fundamental relation between supermassive black holes and their host galaxies," Astrophysical Journal, vol. 539, no. 1, pp. L9-L12, 2000.

[70] K. Gebhardt, R. Bender, G. Bower et al., "A relationship between nuclear black hole mass and galaxy velocity dispersion," Astrophysical Journal, vol. 539, no. 1, pp. L13-L16, 2000.

[71] S. Tremaine, K. Gebhardt, R. Bender et al., "The slope of the black hole mass versus velocity dispersion correlation," Astrophysical Journal, vol. 574, no. 2 I, pp. 740-753, 2002.

[72] J. E. Greene, C. Y. Peng, M. Kim et al., "Precise black hole masses from megamaser disks: black hole-bulge relations at low mass," Astrophysical Journal, vol. 721, no. 1, pp. 26-45, 2010.

[73] J. Kormendy and R. C. Kennicutt, "Secular evolution and the formation of pseudobulges in disk galaxies," Annual Review of Astronomy and Astrophysics, vol. 42, pp. 603-683, 2004.

[74] D. B. Fisher and N. Drory, "Bulges of nearby galaxies with Spitzer: scaling relations in pseudobulges and classical bulges," Astrophysical Journal, vol. 716, no. 2, pp. 942-969, 2010 . 
[75] P. Erwin, J. C. Vega Beltrán, A. W. Graham, and J. E. Beckman, "When is a bulge not a bulge? Inner disks masquerading as bulges in NGC 2787 and NGC 3945," Astrophysical Journal Letters, vol. 597, no. 2 I, pp. 929-947, 2003.

[76] T. H. Jarrett, T. Chester, R. Cutri, S. E. Schneider, and J. P. Huchra, "The 2mass large galaxy atlas," Astronomical Journal, vol. 125, no. 2, pp. 525-554, 2003.

[77] C. M. Carollo, M. Stiavelli, M. Seigar, T. P. De Zeeuw, and H. Dejonghe, "Spiral galaxies with HST/NICMOS. I. Nuclear morphologies, color maps, and distinct nuclei," Astronomical Journal, vol. 123, no. 1, pp. 159-183, 2002.

[78] M. Balcells, A. W. Graham, L. Domínguez-Palmero, and R. F. Peletier, "Galactic bulges from Hubble Space Telescope nearinfrared camera multi-object spectrometer observations: the lack of r1/4 bulges," Astrophysical Journal, vol. 582, no. 2, pp. L79-L82, 2003.

[79] L. Ferrarese, P. Côté, E. D. Bontà et al., "A fundamental relation between compact stellar nuclei, supermassive black holes, and their host galaxies," Astrophysical Journal, vol. 644, pp. 21-24, 2006.

[80] J. Rossa, R. P. Van Der Marel, T. Böker et al., "Hubble space telescope stis spectra of nuclear star clusters in spiral galaxies: dependence of age and mass on hubble type," Astronomical Journal, vol. 132, no. 3, pp. 1074-1099, 2006.

[81] P. Côté, S. Piatek, L. Ferrarese et al., "The ACS Virgo Cluster Survey. VIII. The nuclei of early-type galaxies," Astrophysical Journal, Supplement Series, vol. 165, no. 1, pp. 57-94, 2006.

[82] A. Seth, M. Agüeros, D. Lee, and A. Basu-Zych, "The coincidence of nuclear star clusters and active galactic nuclei," Astrophysical Journal, vol. 678, no. 1, pp. 116-130, 2008.

[83] A. W. Graham and L. R. Spitler, "Quantifying the coexistence of massive black holes and dense nuclear star clusters," Monthly Notices of the Royal Astronomical Society, vol. 397, no. 4, pp. 2148-2162, 2009.

[84] B. M. Peterson and A. Wandel, "Keplerian motion of broadline region gas as evidence for supermassive black holes in active galactic nuclei," Astrophysical Journal, vol. 521, no. 2, pp. L95-L98, 1999.

[85] S. Kaspi, P. S. Smith, H. Netzer, D. Maoz, B. T. Jannuzi, and U. Giveon, "Reverberation measurements for 17 quasars and the size-mass-luminosity relations in active galactic nuclei," Astrophysical Journal, vol. 533, no. 2, pp. 631-649, 2000.

[86] M. Vestergaard, "Determining central black hole masses in distant active galaxies," Astrophysical Journal, vol. 571, no. 2 I, pp. 733-752, 2002.

[87] C. H. Nelson, R. F. Green, G. Bower, K. Gebhardt, and D. Weistrop, "The relationship between black hole mass and velocity dispersion in Seyfert 1 galaxies," Astrophysical Journal, vol. 615, no. 2 I, pp. 652-661, 2004.

[88] C. A. Onken, L. Ferrarese, D. Merritt et al., "Supermassive black holes in active galactic nuclei. II. Calibration of the black hole mass-velocity dispersion relationship for active galactic nuclei," Astrophysical Journal, vol. 615, no. 2 I, pp. 645-651, 2004.

[89] A. J. Barth, J. E. Greene, and C. H. O. Luis, "Dwarf Seyfert 1 nuclei and the low-mass end of the $\mathrm{MBH}-\sigma$ relation," Astrophysical Journal, vol. 619, no. 2, pp. L151-L154, 2005.

[90] M. C. Bentz, B. M. Peterson, H. Netzer, R. W. Pogge, and M. Vestergaard, "The radius-luminosity relationship for active galactic nuclei: the effect of host-galaxy starlight on luminosity measurements. II. the full sample of reverberationmapped agns," Astrophysical Journal, vol. 697, no. 1, pp. 160 181, 2009.
[91] H. Falcke and P. L. Biermann, "The jet-disk symbiosis. I. Radio to X-ray emission models for quasars," Astronomy and Astrophysics, vol. 293, pp. 665-682, 1995.

[92] S. Heinz and R. A. Sunyaev, "The non-linear dependence of flux on black hole mass and accretion rate in core-dominated jets," Monthly Notices of the Royal Astronomical Society, vol. 343, no. 3, pp. L59-L64, 2003.

[93] E. Körding, H. Falcke, and S. Corbel, "Refining the fundamental plane of accreting black holes," Astronomy and Astrophysics, vol. 456, no. 2, pp. 439-450, 2006.

[94] J. L. Turner and P. T. P. Ho, "Bright radio continuum emission from star formation in the cores of nearby spiral galaxies," Astrophysical Journal, vol. 421, no. 1, pp. 122-139, 1994.

[95] N. Smith, J. M. Silverman, R. Chornock et al., "Coronal lines and dust formation in SN 2005ip: not the brightest, but the hottest type IIn supernova," Astrophysical Journal, vol. 695, no. 2, pp. 1334-1350, 2009.

[96] D. Pooley, S. Immler, and A. V. Filippenko, "Chandra observation of SN 2005kd: very luminous and hard X-ray emission," The Astronomer's Telegram, vol. 1023, p. 1, 2007.

[97] S. Immler, P. J. Brown, P. Milne et al., "X-ray, UV, and optical observations of supernova 2006bp with Swift: detection of early X-ray emission," Astrophysical Journal, vol. 664, no. 1 I, pp. 435-442, 2007.

[98] S. Komossa, H. Zhou, A. Rau et al., "NTT, spitzer, and chandra spectroscopy of SDSSJ095209.56+214313.3: the most luminous coronal-line supernova ever observed, or a stellar tidal disruption event?" Astrophysical Journal, vol. 701, no. 1, pp. 105-121, 2009.

[99] S. D. Van Dyk, K. W. Weiler, R. A. Sramek, and N. Panagia, "SN 1988Z: the most distant radio supernova," Astrophysical Journal, vol. 419, no. 2, pp. L69-L72, 1993.

[100] C. L. Williams, N. Panagia, S. D. Van Dyk, C. K. Lacey, K. W. Weiler, and R. A. Sramek, "Radio emission from SN 1988 Z and very massive star evolution," Astrophysical Journal, vol. 581, no. 1 I, pp. 396-403, 2002.

[101] P. Chandra, V. V. Dwarkadas, A. Ray, S. Immler, and D. Pooley, "X-rays from the explosion site: 15 years of light curves of SN 1993J," Astrophysical Journal, vol. 699, no. 1, pp. 388-399, 2009. 

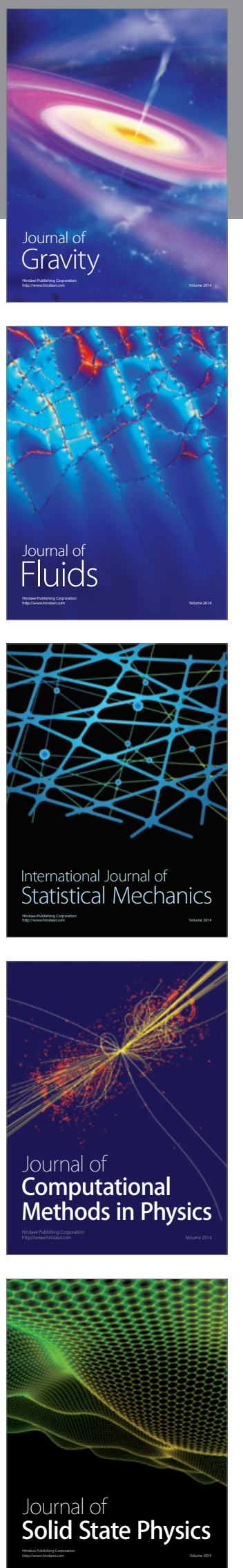

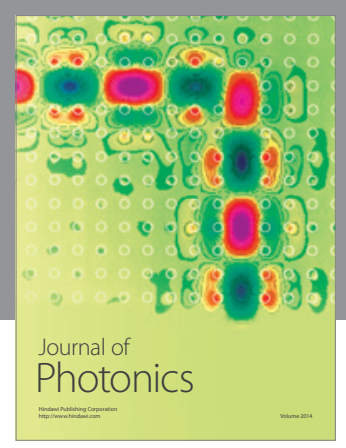

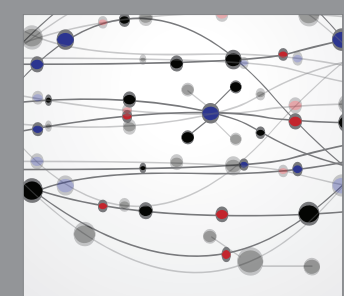

The Scientific World Journal
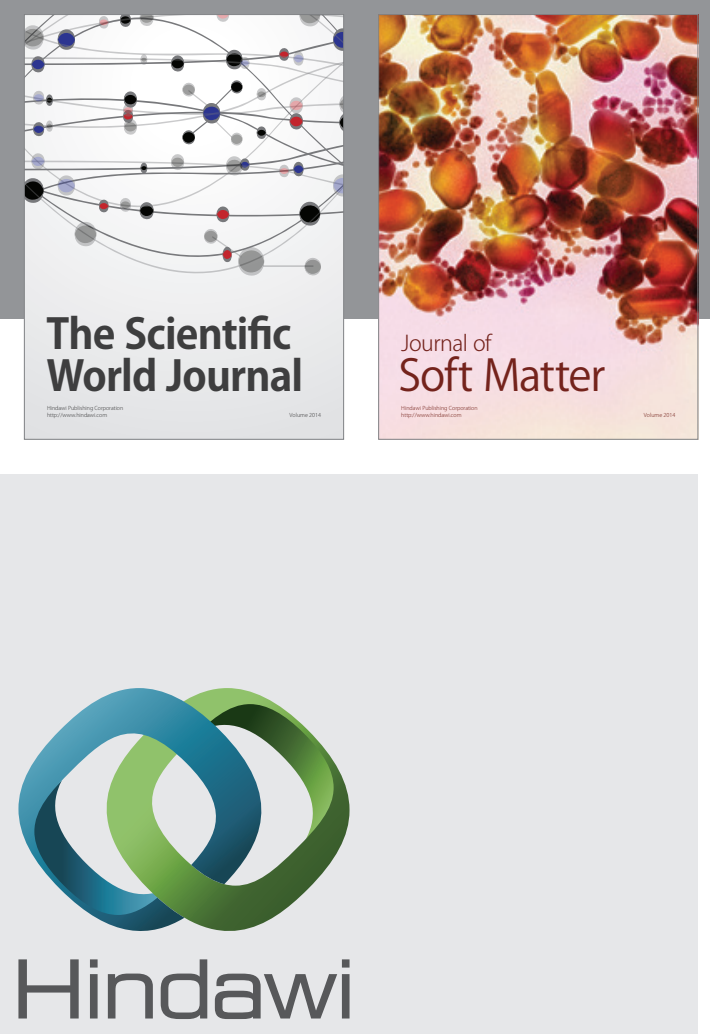

Submit your manuscripts at

http://www.hindawi.com
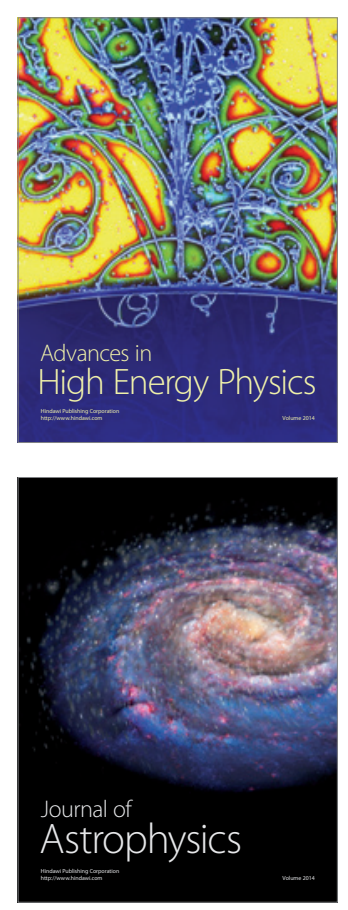
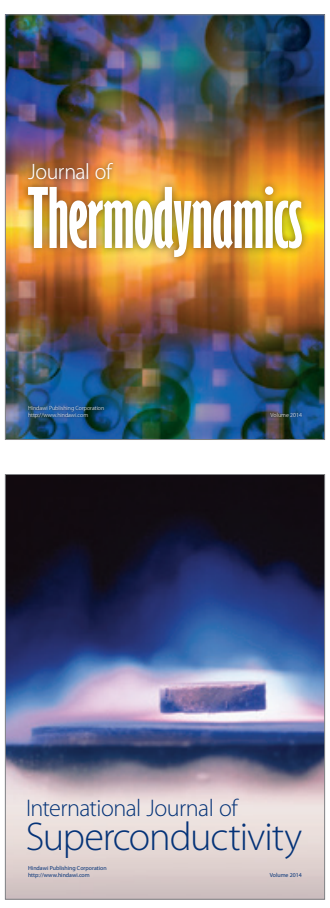
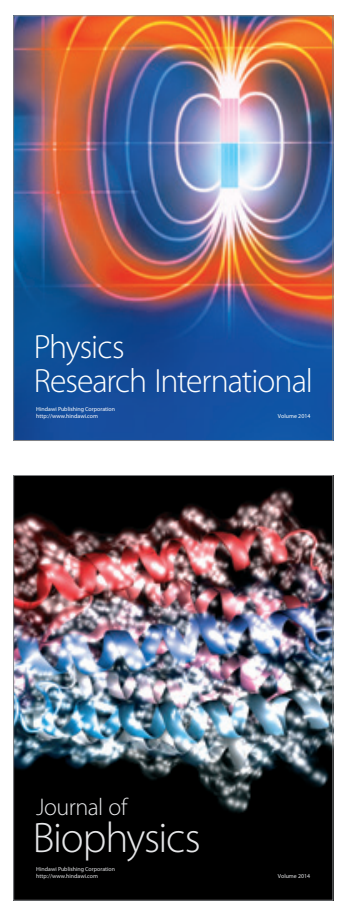
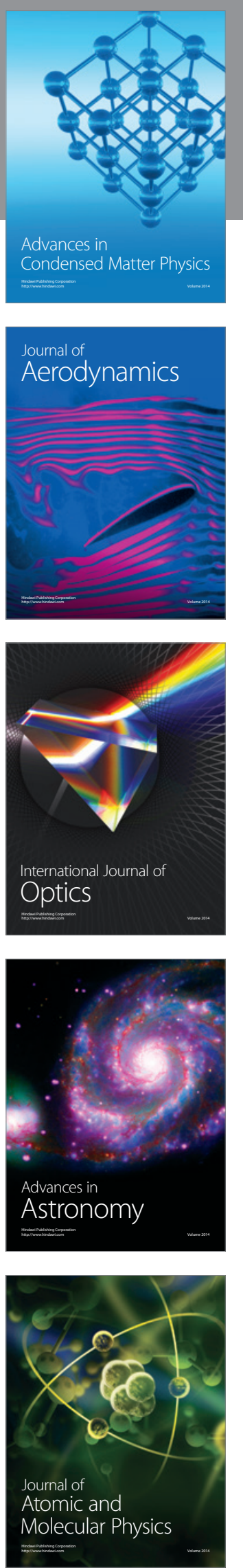